\title{
Article
}

\section{An siRNA-guided Argonaute protein directs RNA Polymerase V to initiate DNA methylation}

Meredith J. Sigman ${ }^{1,2}$, Kaushik Panda ${ }^{2}$, Rachel Kirchner ${ }^{1, \S}$, Lauren L. McLain², Hayden Payne ${ }^{2}$, John Reddy Peasari ${ }^{2,3}$, Aman Y. Husbands ${ }^{1}$, R. Keith Slotkin ${ }^{2,4^{*}}$ and Andrea D. McCue ${ }^{1,2}$

1 Department of Molecular Genetics, The Ohio State University, Columbus, Ohio, 43210, USA

2 Donald Danforth Plant Science Center, St. Louis, Missouri, 63132, USA

3 Bioinformatics and Computational Biology Program, Saint Louis University, St. Louis, Missouri, 63108, USA

4 Division of Biological Sciences, University of Missouri, Columbia, Missouri, 65211, USA

$\S$ Present Address: Medical Scientist Training Program, University of Wisconsin, Madison, Wisconsin, 53705, USA

* Corresponding Author, kslotkin@danforthcenter.org 


\section{Article}

Keywords: RNA-directed DNA methylation (RdDM); small interfering RNA (siRNA); RNA Polymerase V; Argonaute; epigenetics, DNA methylation

\section{Abstract}

In mammals and plants, cytosine DNA methylation is essential for the epigenetic repression of transposable elements and foreign DNA. In plants, DNA methylation is guided by small interfering RNAs (siRNAs) in a self-reinforcing cycle termed RNA-directed DNA methylation (RdDM). RdDM requires the specialized RNA Polymerase V (Pol V), and the key unanswered question is how Pol $V$ is first recruited to new target sites without preexisting DNA methylation. We find that Pol $\mathrm{V}$ follows and is dependent upon the recruitment of an AGO4-clade ARGONAUTE protein, and any siRNA can guide the ARGONAUTE protein to the new target locus independent of preexisting DNA methylation. These findings reject long-standing models of RdDM initiation and instead demonstrate that siRNA-guided ARGONAUTE targeting is necessary, sufficient and first to target Pol $V$ recruitment and trigger the cycle of RdDM at a transcribed target locus, thereby establishing epigenetic silencing. 
Chromatin marks segregate genomes into expressed domains and regions that remain transcriptionally silenced. In mammals and plants, DNA methylation provides information such as which regions are transposable elements (TEs) and should not be expressed (reviewed in ${ }^{1}$ ), while integrated transgenes are often subject to this regulation as well ${ }^{2}$. Most studies have focused on how DNA methylation is epigenetically maintained, resulting in heritable transcriptional repression. However, how DNA methylation is initially established at individual loci is less understood.

In both plants and mammals, DNA methylation is targeted via the action of small RNAs (piRNAs in mammals) ${ }^{3,4}$. Specifically in plants, small interfering RNAs (siRNAs) are produced from TEs, viruses and transgenes, targeting them for RNA-directed DNA methylation $(\operatorname{RdDM})\left(\right.$ reviewed in $\left.{ }^{5}\right)$. RdDM is a feed-forward cycle that reinforces DNA methylation and results in epigenetic transcriptional repression. The mechanism of $\mathrm{RdDM}$ is split between an upstream siRNA-generating phase and a downstream chromatin-linked phase. In the upstream phase, siRNAs are generated from either RNA Polymerase IV (Pol IV)- or Pol II-derived transcripts. In the downstream phase, these siRNAs are incorporated into one of the closely related ARGONAUTE proteins AGO4, AGO6 or AGO96 ${ }^{6}$. Base complementarity between the siRNA and a chromatin-linked nascent transcript results in recruitment of the de novo DNA methyltransferases DRM1 and DRM2 ${ }^{7}$. The nascent transcript is produced by RNA Polymerase V (Pol V), which provides the scaffold for AGO/siRNA complex interaction ${ }^{8,9}$. Pol IV and Pol V are derived from Pol II, as subunits of these holoenzymes duplicated early in plant evolution and subfunctionalized into their respective roles in siRNA biogenesis (Pol IV) and scaffolding RNA production $(\mathrm{Pol} \mathrm{V})^{10}$.

Pol $V$ is continually recruited to RdDM loci through its interaction with the proteins SUVH2 and SUVH9, which bind existing cytosine DNA methylation ${ }^{11,12}$. However, how Pol V is recruited to new unmethylated DNA to first trigger RdDM and establish chromatin marks is not understood. One popular model in the literature proposes that, in the absence of Pol V, a Pol IIderived transcript acts as the scaffold and can set the initial round of DNA methylation ${ }^{13,14}$. A second model suggests that Pol $V$ ubiquitously surveys the genome at low levels, and the first round of RdDM occurs upon addition of siRNAs. This 'Pol V surveillance' model is supported by a recent publication demonstrating that $\mathrm{Pol} V$ is present at a second set of loci that do not undergo RdDM because they lack siRNAs ${ }^{15}$. In both models, after the first round of methylation, Pol V would then be recruited through the activity of SUVH2/SUVH9, leading to the positive feed-forward loop of RdDM. Neither of the two models have been examined in the context of 
true 'de novo' silencing as in ${ }^{16}$, and this leaves a formidable gap in our understanding of how the first round of DNA methylation is established.

Since RdDM is a self-reinforcing cycle, it is impossible to study the first round for endogenous regions of the genome that are already engaged. To address this, we developed an approach that interrogates de novo DNA methylation of newly transformed transgene DNA, so that no preexisting chromatin marks are guiding RdDM. We used this system to address a critical question in plant epigenetics: how is Pol V first recruited to new unmethylated sites for the first round of RdDM? Our findings demonstrate that, contrary to previous models, AGO4clade proteins precede Pol V recruitment to new targets of RdDM. This mechanism provides the missing link between unmethylated DNA and the initiation of chromatin modification towards epigenetic silencing.

\section{Results}

A transgenic system to investigate the first round of DNA methylation

The expression of TE-derived sequences reproducibly triggers $\operatorname{RdDM}^{17,18}$. To study the initiation of DNA methylation, we recreated the '35S:EVD' transgene consisting of a broadly expressed promoter driving the full-length coding sequence of the Arabidopsis Evadé TE ${ }^{19}$. This transgene was stably integrated into wild-type Columbia (wt Col) plant genomes, and transgene silencing in the first-generation (T1) transformants was assayed for all experiments. To

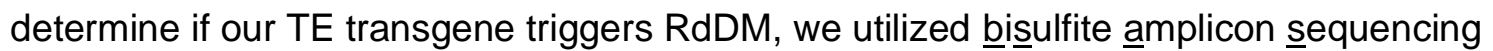
$(B S A S)^{20}$, a technique with high sequencing depth. In a side-by-side comparison, the average methylation remains the same for BSAS, Sanger sequencing and whole-genome sequencing techniques at target loci after conversion with sodium bisulfite, but the resolution of the data with BSAS is superior ( 3800 average coverage)(Supplemental Figure 1). When BSAS and small RNA sequencing were applied to T1 35:EVD plants, we found that siRNAs are generated from the transgene (Supplemental Figure 2), which results in high levels of de novo DNA methylation (Figure $1 \mathrm{~A}$ and Supplemental Figure 2). Furthermore, although the coding sequence in 35S:EVD is an exact copy of the Evadé element EVD5 from the Arabidopsis genome (At5TE20395), transgene methylation is not influenced in trans by siRNAs from the endogenous EVD elements (Supplemental Figure 2).

\section{SUVH2/SUVH9-independent Pol V function}

Pol II has been proposed to substitute for Pol V during the first round of RdDM. To test this, we interrogated the methylation of T1 35S:EVD in pol $V$ mutants (nrpE1 subunit mutation). 
We found that Pol V is necessary to trigger RdDM, as plants lacking Pol V have only background levels of methylation similar to plants lacking de novo DNA methyltransferase activity (in the $d r m 1 / 2$ double mutant), and similar to the non-conversion rate during sodium bisulfite treatment (Figure 1A)(non-conversion rates calculated in Supplemental Figure 1). BSAS affords increased data resolution ${ }^{20}$, which reveals that the low level of DNA methylation in pol $V$ mutants exists as sporadic unconverted cytosines (stochastic), rather than as consecutive runs of methylated cytosines (indicative of DRM1/DRM2 activity in RdDM)(Supplemental Figure 1). We assayed RdDM strength by quantifying these strings of consecutive methylated bases (see Methods and controls in Supplemental Figure 1), which takes advantage of the large number of reads generated by BSAS to individually score each read, rather than averaging the data as in other methylation analysis methods. Using this improved methodology, we found no evidence of RdDM activity in pol V mutants (Figure 1B). Our data demonstrates that, contrary to the Pol II substitution model, Pol V is essential to initiate the first round of DNA methylation.

The only known mechanism for directly recruiting Pol $V$ is through the DNA methylbinding proteins SUVH2/SUVH9 ${ }^{11,12}$. We found that the DNA methylation of 35S:EVD in plants lacking both SUVH2 and SUVH9 does not phenocopy the total loss of RdDM in pol Vor drm1/2 mutants (Figure 1A). Rather, suvh2/suvh9 double mutants have an intermediate level of DNA methylation and RdDM strength (Figure 1A-B), suggesting that these proteins function to amplify DNA methylation levels rather than trigger RdDM at new locations. This demonstrates that there is a mechanism other than SUVH2/SUVH9 for the first round function of Pol V at an unmethylated target locus.

\section{AGO4-clade proteins are required for the first round of RdDM}

To determine what other pathway components are essential for the first round of RdDM, we transformed 35S:EVD into a series of known DNA methylation, RdDM and RNAi mutants. As expected, 35S:EVD methylation and siRNA production are not dependent on DNA methyltransferases that propagate DNA methylation during DNA replication (maintenance methyltransferases)(Supplemental Figure 3). Rather, its methylation is dependent on at least one of the closely related AGO4-clade proteins (AGO4, AGO6 or AGO9 in the ago4/6/9 triple mutant)(Figure 2A, Supplemental Figure 4). Because AGO2 has a published role in $\mathrm{RdDM}^{21}$, we additionally tested ago2 mutants and found only partial reductions in 35S:EVD 21-22 nt siRNAs, DNA methylation and RdDM strength, suggesting it has a secondary role during the initiation of DNA methylation (Supplemental Figure 5). Furthermore, we used immunoprecipitation followed by small RNA sequencing and found that 35S:EVD-derived siRNAs are enriched in AGO4 
protein complexes (Figure 2C, controls in Supplemental Figure 6), demonstrating the direct role of AGO4 in the RdDM of 35S:EVD.

SiRNA biogenesis is not dependent upon Pol V, DRM1/DRM2 or AGO4/AGO6/AGO9 (Figure 2B, Supplemental Figure 4), confirming that these proteins function downstream of siRNA production during the chromatin-linked phase of RdDM. Instead, mutations in the downstream factors Pol V, DRM1/DRM2 and AGO4-clade proteins surprisingly have increased siRNA accumulation (Figure 2B). We found that this increase in siRNAs positively correlates with the level of Pol II transcription of 35S:EVD in drm1/2 and ago4/6/9 mutant (Figure 2D), and is inversely correlated with the level of the transcriptionally-repressive histone mark H3K9me2 (Figure 2E). This demonstrates that in mutants of RdDM downstream machinery (pol V, drm1/2, ago4/6/9), without DNA methylation and H3K9me2, 35S:EVD expression is uninhibited, generating more transcription that leads to increased siRNA production. Additional regions of the endogenous genome also generate more siRNAs in pol V, drm1/2 and ago4/6/9 mutants (Supplemental Figure 7), suggesting that a lack of downstream RdDM function broadly leads to enhanced Pol II transcription and increased siRNA production.

\section{Pol Il-derived DCL-processed siRNAs trigger RdDM}

Our data suggests that siRNA production from 35S:EVD is from Pol II, since siRNA accumulation is not lost in pol IV or pol $V$ mutants (Figure 2B) and the abundance of siRNAs (Figure 2B) positively correlates with the level of Pol II transcription of 35S:EVD (Figure 2D). In plants, siRNA sizes and categories are determined by the specific DICER family (DCL) protein that produces them ${ }^{22}$. Our data refutes an existing model based on the identical 35S:EVD transgene that RdDM begins only when DCL2 and DCL4 are overwhelmed with doublestranded RNA substrate, thus activating DCL3 to generate $24 \mathrm{nt}$ siRNAs ${ }^{19}$. Instead, we find that dcl2/4 double mutants result in a $\sim 50 \%$ reduction in DNA methylation (Figure $2 \mathrm{~F}$ ) rather than being hyper-methylated as previously posited ${ }^{19}$. Further, DNA methylation is not entirely dependent upon the presence of 24 nt siRNAs, as dc/3 mutants that lack 24 nt siRNAs (Figure $2 \mathrm{G}$ ) do not lose all methylation (Figure $2 \mathrm{~F}$ )(as in $\left.{ }^{16}\right)$. Additionally, DNA methylation still persists in $d c / 2 / 3 / 4$ triple mutants, where only DCL1-dependent $21 \mathrm{nt}$ siRNAs remain (Figure $2 \mathrm{~F}-\mathrm{G}$ ). These data indicate that 21, 22 and $24 \mathrm{nt}$ siRNAs are all sufficient to trigger the initiation of RdDM. DNA methylation is nearly absent only when siRNAs are severely reduced in $d c / 1 / 2 / 3 / 4$ quadruple mutants (Figure 2F-G). Therefore, the initiation of DNA methylation is fully dependent on the presence of Pol II-derived DCL-processed siRNAs in a size-independent manner. 
Pol $V$ requires an AGO4-clade protein for localization during the initiation of RdDM

Pol V is essential to establish RdDM (Figure 1), and therefore we aimed to understand how Pol $\mathrm{V}$ is recruited to a locus in the absence of preexisting DNA methylation. We reanalyzed published Pol V chromatin immunoprecipitation (ChIP) and RNA immunoprecipitation (RIP) data ${ }^{12,9}$ to determine whether Pol V is present (ChIP) and transcribing (RIP) at low levels throughout the genome. Our re-analysis centered on using mitochondrial genes as additional negative baseline controls, as Pol V subunits are located exclusively in the nucleus ${ }^{23,24}$. We find that Pol V signal is detected at non-RdDM nuclear loci only at the same rate as the mitochondrial negative control genes (Figure $3 \mathrm{~A}$ ). Therefore, rather than patrolling all loci at low levels, Pol $\mathrm{V}$ is actively recruited to its new target sites for the initiation of RdDM.

We identified three mutant combinations of downstream RdDM factors that completely lose the ability to target T1 35S:EVD for RdDM ( $p o l ~ V$, drm1/2 and ago4/6/9)(Figure 2). To test the requirements of Pol V recruitment to new loci, we performed Pol V (subunit NRPE1) ChIP in these mutant backgrounds in the T1 generation of 35S:EVD plants. We found that the Pol V protein still accumulates in each of these mutant backgrounds (Supplemental Figure 7) and is recruited in the first generation to the TE transgene (Figure $3 \mathrm{~B}$ ). Importantly, this recruitment is not dependent on existing DNA methylation, as Pol V recruitment still occurs in the $d r m 1 / 2$ mutant (Figure 3B) that lacks DNA methylation (Figure 1). Therefore, this system provides the ability to dissect the methylation-independent recruitment of Pol V. Importantly, we found that Pol V is not recruited for the first round of RdDM in the ago4/6/9 triple mutant (Figure 3B). This was surprising, as the prevailing dogma suggests that Pol V presence and transcription occurs first and is required to position AGO4-clade proteins at the chromatin target ${ }^{25}$. Conversely, our data demonstrates that AGO4-clade proteins are required to localize Pol $V$ during the initiation of RdDM.

AGO4-clade proteins are directed to their targets by the complementarity of their incorporated siRNAs ${ }^{26}$. To determine if there are regions of the endogenous genome where Pol V is positioned independently of SUVH2/SUVH9 and instead based on AGO4/siRNAs, we first identified regions of the genome where Pol V occupancy is only partially-dependent on the methylation-dependent SUVH2/SUVH9 mechanism (left, Figure 3C). These 113 regions retain siRNA accumulation in suvh2/suvh9 and ago4/6/9 mutants (Figure 3D). Conversely, the 1455 regions of the genome where Pol V recruitment is fully dependent on SUVH2/SUVH9 tend to have reduced siRNA accumulation in suvh2/suvh9 mutants (Figure 3D). These data suggest that when the SUVH2/SUVH9 recruitment method is absent, the continual production of siRNAs is necessary for Pol V occupancy at a small number of loci in the Arabidopsis genome. 
AGO4 can interact with new sites of RdDM independent of Pol V and DNA methylation

We aimed to order the events of recruitment at the chromatin that result in the first round of RdDM. Since Pol V recruitment to 35S:EVD is dependent on a protein of the AGO4-clade (Figure 3), we aimed to determine if the converse was true: Is AGO4's interaction with chromatin dependent on Pol V? Our data supports this idea that there are a small number of regions of the endogenous Arabidopsis genome where AGO4 interacts with target chromatin loci independent of a Pol V-derived scaffolding transcript. Since AGO4 targeting is dependent on siRNA complementarity, we began by identifying loci that continue to produce 23-24 nt siRNAs in a pol $V$ mutant (Figure 4A). We overlapped these 4246 Pol V-independent siRNA loci with 820 previously-identified AGO4 bound loci ${ }^{27}$, resulting in 91 testable AGO4-bound regions of the genome that do not lose siRNAs (Figure 4B). 63 of these testable AGO4-bound regions $(69 \%)$ retain AGO4 occupancy in the pol V mutant (Figure 4C), demonstrating AGO4 recruitment without Pol V.

To determine if AGO4 is localized to the 35S:EVD transgene during the initiation of RdDM, we performed AGO4 ChIP on T1 35S:EVD plants. As a control, we confirmed that the AGO4 protein is present in the various mutants we tested (Supplemental Figure 7). In the ChIP experiment, we detected AGO4 at 35S:EVD during the initiation of RdDM in wild-type plants, as well as in pol V, suvh2/suvh9 and drm1/2 mutants (Figure 4D-E). The level of AGO4 at 35S:EVD in pol V and suvh2/suvh9 mutants is not as high as in wt Col plants, but is nonetheless significantly higher than the negative control AXS2 gene and the IgG negative control (Figure 4D-E). In addition, the AGO4 ChIP signal in the $d r m 1 / 2$ mutant may be higher due to the increased siRNAs produced in this mutant (Figure 2B). We conclude that AGO4 is directed to the target transgene without the requirement of a Pol V scaffolding transcript or DNA methylation, and is independent of the known SUVH2/SUVH9 recruitment mechanism of Pol V (Figure 4E), placing AGO4 interaction with the target locus before and not reliant upon Pol V activity.

\section{siRNAs are sufficient to direct Pol V function}

Since AGO4 can be recruited to a new target locus independent of Pol V, we aimed to determine if siRNAs are sufficient to direct Pol V activity and the first round of RdDM. Our 35S:EVD transgene system cannot address this question, since the source locus of the siRNAs and the target of Pol V action are the same (cis-acting). Instead, we generated a trans-acting two component system to uncouple siRNA production from Pol V recruitment. SiRNAs are 
generated from an inverted repeat transgene that targets an unmethylated endogenous gene in trans (Figure $5 \mathrm{~A}$ ) (as in ${ }^{28,29,30}$ ). The endogenous gene we targeted (At3G12210) encodes a broadly expressed DNA-binding protein that does not produce siRNAs (Figure 5B), is not methylated (Figure 5C), and is not bound by Pol V (Figure 3A) in wt Col plants. We named this uncharacterized gene SQUEAKY1 (SQK1). Upon addition of the SQK1 inverted repeat transgene ('SQK1-IR'), 21, 22 and 24 nt siRNAs accumulate from the hairpin region (Figure $5 \mathrm{~B}$ ), and these siRNAs function in trans to direct RdDM to the SQK1 endogenous gene (Figure 5C-D). In a pol $V$ mutant, siRNAs are still generated from the IR transgene (Figure 5B), but RdDM does not occur (Figure 5C-D), again demonstrating that Pol V acts downstream of siRNA production and is essential for RdDM. As with 35S:EVD, the initiation of RdDM is fully dependent on an AGO4-clade protein (Figure 5C-D). Taken together, these data demonstrate that the production of new siRNAs and the presence of an AGO4-clade protein are sufficient to target Pol V-dependent RdDM to a new non-transgenic locus independent of existing methylation.

\section{Pol Il expression is necessary for permitting the first round of RdDM}

While Pol II cannot substitute for Pol V (Figure 1), we have identified that Pol II does play a role in making the target locus receptive to the first round of RdDM. While investigating the targeted methylation of the SQK1 gene (Figure 5), we observed that the $\mathrm{CHH}$ methylation (indicative of RdDM) was not distributed evenly across the region targeted by siRNAs. Instead, methylation peaks upstream of the transcriptional start site (TSS) near the proximal SQK1 promoter (Figure 6A). This pattern of methylation does not correlate with the abundance of siRNAs across this region (Figure 6A), suggesting that the observed methylation pattern is based on the SQK1 promoter activity. To test the role of Pol II expression during the first round of RdDM, we used Cas9 to generate a $1298 \mathrm{bp}$ deletion of the SQK1 promoter (sqk1-1)(shown in Figure 6A). This severely reduces, but does not completely eliminate SQK1 expression (Figure 6B). Where on the sqk1-1 allele Pol II initiates and the direction of transcription is not known. When the homozygous sqk1-1 mutation is combined with the SQK1-IR, although the siRNAs still target sqk1-1, methylation is significantly reduced (Figure 6A,C). In a separate test, we generated a second IR targeting system (as in Figure 5A) for a different gene that has a specific and limited developmental expression pattern (Supplemental Figure 8). Our data for both two-component IR systems (Figure 6A-C and Supplemental Figure 8) demonstrates that the strength of RdDM positively correlates with the level of Pol II expression at the target locus. 
To decisively test the necessity of Pol Il expression in the first round of RdDM, we created two new EVD transgenes that are definitively either expressed or not. These include the 'EVD-only' (EVD coding sequence with no promoter) and 'T3A-EVD' transgenes (T3A terminator directly 5' of the EVD coding sequence, to ensure no read-through Pol II transcription)(Figure 6D). qRT-PCR of EVD transgenes confirms that only 35S:EVD has appreciable mRNA accumulation (Figure 6E), although the expression levels generated by the $35 \mathrm{~S}$ promoter are highly variable due to the nature of $\mathrm{T} 1$ transgenesis ${ }^{31}$. We confirmed that RdDM of 35S:EVD is dependent on Pol II expression, as only the expressed transgene version is targeted for methylation (Figure 6F, biological replicates in Supplemental Figure 8). The key test was when we combined expressed and unexpressed EVD transgenes in the same genome. We found that whenever the expressed 35S:EVD transgene is present, it produces abundant siRNAs (Figure 2B and Figure 6G lanes 1, 4, 5), which are incorporated into AGO4 (Supplemental Figure 6) and drive RdDM to 35S:EVD itself (Figure 1 and Figure 6H lanes 1, 4, 5). When a second promoterless EVD transgene is introduced into the same plant genome that has 35S:EVD, these unexpressed transgenes do not become methylated (Figure 6H lanes 4-5, biological replicates in Supplemental Figure 8). These double-transgenic plants have EVD siRNAs that are incorporated into AGO4 and are competent to perform RdDM (evidenced by the fact that 35S:EVD is targeted by RdDM in the same plant (Figure 6G lanes 4-5)). The only difference as to why one transgene is methylated and the other is not is the Pol II activity at the target transgene. Therefore, Pol II transcription is not sufficient to substitute for the absence of Pol V, but Pol II activity is necessary to make a locus receptive to the first round of RdDM.

\section{Pol Il's role in the initiation of RdDM}

We aimed to identify the exact function of Pol II during the first round of RdDM. Pol II could be involved in producing a scaffolding transcript for AGO/siRNA interaction. Alternatively, AGO/siRNA complexes are known to interact with single-stranded DNA ${ }^{32}$. Pol II's function at the target locus could be to open the double-stranded structure of the DNA, allowing for the AGO/siRNA complex to base pair with single-stranded DNA, which fits the theory that RdDM is focused at DNA replication forks ${ }^{33}$. To separate these models, we attempted to increase the RdDM of the low-expressed sqk1-1 allele by targeting dCas9 to this locus. We used a gRNA to target dCas9 and R-loop formation ${ }^{34}$ to the homozygous sqk1-1 locus in the presence of the SQK1-IR (see gRNA location in Figure 6A). This did not result in higher methylation (Figure $6 A, C)$ as would be expected if DNA opening was the RdDM function of Pol II at the target locus. Instead, methylation decreased, as though dCas9 interferes with the low level of Pol II 
transcription at this locus. Since this dCas9 experiment provided negative results, several alternative interpretations exist. However, this experiment suggests that the function of Pol II at a new RdDM target locus is likely to generate the RNA transcript that acts in AGO/siRNA complementary base pairing.

\section{Discussion}

The roles of RNA Polymerase IV and V at silenced TE fragments undergoing the selfreinforcing cycle of RdDM are well described ${ }^{35}$. However, the specific roles of these polymerases and Pol II during the initiation phase of TE and transgene silencing have remained enigmatic. Here we dissect polymerase function using newly integrated transgenes. This strategy permits the discrimination of the first round of RdDM from those already engaged in the RdDM cycle.

We find that Pol V is required for all de novo RdDM, and Pol II cannot substitute for this function. Our data refutes the model whereby Pol II transcripts are able to initiate the first round of DNA methylation. The essential function of Pol V during the initiation of RdDM correlates with the recent finding that Pol V is a key factor in the evolutionary repression of TEs in the Arabidopsis lineage ${ }^{36}$. Our data also refutes the 'Pol V surveillance' model, whereby Pol V produces scaffolding transcripts that, when an siRNA is present, will trigger the first round of methylation. It has been recently demonstrated that the artificial tethering of a Pol V-recruitment factor to a genomic locus triggers $\mathrm{RdDM}^{37}$. Therefore, if Pol V surveilled everywhere, we would expect to observe spurious low-level methylation across the genome, which is not detected, even in DNA glycosylase mutants that fail to remove DNA methylation from the genome ${ }^{38}$. Although our data refutes the Pol $V$ surveillance model, our findings agree with the point that siRNAs are the key determinant to target new $\operatorname{RdDM}^{15}$.

Our data demonstrates a methylation-independent mechanism for recruitment of Pol V to a new target locus. Previous reports suggested that Pol V is recruited independently of AGO4 and siRNAs, and RdDM would occur only when both Pol V and AGO4 were independently recruited to a locus ${ }^{39}$. Our results suggest a more linear ordered pathway of recruitment, beginning with AGO4 directed by siRNAs. We find that AGO4 is recruited to a new site of RdDM independent of and before Pol V and DNA methylation, and AGO4-clade proteins are themselves guided by the complementarity of the incorporated siRNA. We propose an siRNAdirected pathway of Pol $\mathrm{V}$ recruitment in Figure 7.

Pol ll's function during the first round of RdDM is two-fold. First, it is necessary to produce the raw transcripts for siRNA production (step 1, Figure 7). During the self-reinforcing 
cycle of RdDM at endogenous silenced TEs, this function is taken over by Pol IV. However without existing heterochromatic marks to recruit Pol IV, the new region must be transcribed by Pol Il to generate siRNAs in cis or siRNAs provided from a separate locus in trans. We find that in cases where Pol II is generating siRNAs, mutations in the downstream RdDM factors (such as pol $V, d r m 1 / 2$ and ago4/6/9) result in the lack of DNA and H3K9 methylation, leading to more Pol II expression and more substrate for the higher accumulation of siRNAs (Figure 2). Once the raw transcripts are produced by Pol II, we find that they can be processed by any DCL protein into 21-24 nt siRNAs (step 2, Figure 7). All of these siRNAs sizes, generated by any of the four Arabidopsis DCL proteins, are capable and sufficient to target the first round of RdDM. Our data agrees with the 'saturation' model whereby during the initiation of RdDM all available DCL proteins function to process the large volume of Pol II transcripts, producing 21, 22 and 24 nt siRNAs that are all capable of guiding $\operatorname{RdDM}^{16}$. These siRNAs are loaded into an AGO4clade protein (step 3, Figure 7), as RdDM function was abolished in the ago4/6/9 triple mutant.

Second, Pol II action is required downstream of siRNA production. Even when complementary siRNAs and AGO4 are present, the first round of RdDM is dependent upon Pol II activity at both transgenes and endogenous genes (Figure 6). Our data suggests that the role of Pol II at the target locus is to generate the first scaffolding transcript for AGO/siRNA interaction (step 4, Figure 7). This is supported by the protein-protein interaction of the Pol V recruitment factor RDM1 with Pol II, AGO4 and DRM2 ${ }^{24}$. This function of Pol II suggests that it retains some of its ancestral ability to generate scaffolding transcripts, which has otherwise been subfunctionalized and relegated to Pol V. Even though the Pol II transcript interaction with the AGO/siRNA complex does not result in methylation, it is necessary for the recruitment of Pol V (step 5, Figure 7) and subsequent RdDM (step 6, Figure 7). A recent study demonstrated that Pol II's C-terminal domain can act to recruit more Pol II protein ${ }^{40}$, and during the initiation of RdDM Pol II may act in a similar manner to recruit Pol V, target RdDM and result in the establishment of epigenetic inheritance.

\section{Methods}

Plant growth and lines

Arabidopsis thaliana plants were grown at $22^{\circ} \mathrm{C}$ on Pro-Mix FPX soil in Conviron MTPS120 growth chambers with 16-hour $200 \mu \mathrm{mol} / \mathrm{m}^{2} / \mathrm{s}$ light. The specific alleles of all mutants are shown in Supplemental Table 1. Inflorescence (flower buds, stage 1-12) tissue was used for all experiments unless otherwise noted. All transgenic material is the first generation (T1) after integration of the T-DNA into the genome unless otherwise stated. All transgenic lines were 
stably integrated and produced by the Agrobacterium-mediated floral dip method and subsequent selection for Basta- or Hygromycin-resistant plants. Biological replicates are nonoverlapping pools of tissue collected from T1 transgenic plants. Double EVD transgenic lines (Figure 6G-H) were generated by floral dipping T2 Basta-resistant 35S:EVD plants with Hygromycin-resistant EVD-only and T3A-EVD transgenes. Screening and genotyping in the next generation resulted in T3 35S:EVD + T1 EVD-only or T1 T3A-EVD plants. To create the $d c / 1 / 2 / 3 / 4$ 35S:EVD transgenic plants, we transformed a line that was homozygous $d c / 2 / 3 / 4$ and heterozygous for the $d c / 1-9$ allele. Resulting transformants were genotyped for DCL1, and divided into those that were homozygous mutants and those that were DCL1 heterozygotes.

\section{Transgene Generation}

Transgenes were generated from primers listed in Supplemental Table 1. The EVD coding sequence (At5TE20395) was directly amplified from wt Col DNA using primers containing appropriate plasmid homology for In-Fusion Cloning (Clontech) into their respective digested binary vector backbones. Both pB2GW7 (Basta selection) and pH2GW7 (Hygromycin selection) digested with Spel/HindIII were used for 35S:EVD, as some mutants that were transformed with this transgene already contain a Basta resistance cassette. pH2GW7 digested with Sacl/HindIII was used for EVD-only. For T3A-EVD, the T3A terminator and EVD coding sequence were separately amplified and then joined together using overlapping PCR before In Fusion Cloning into pH2GW7 digested with Sacl/HindIII.

The inverted repeat regions of transgenes were synthesized by Thermo-Fisher and cloned into the binary vector pEG100 via Spel/Xhol restriction digest followed by ligation using T4 DNA Ligase (NEB). The SQK1 inverted repeat contains bases AtChr3 3895083:3894528 (order reversed to prevent open reading frame) followed by the PDK intron and then AtChr3 33894528:3895083 (resulting plasmid named pMJS064). The RHD6 inverted repeat was fashioned similarly containing bases AtChr1 24795653:24795105. See Supplemental Table 1 for inverted repeat sequences. A Hygromycin-resistant version of each of these transgenes was created by amplifying the $2 X 35 S$ promoter: Hygromycin b phosphotransferase: $35 S$ terminator cassette from the pDIRECT_21B vector using primers from Supplemental Table 1 to add Mlul and Sacl restriction sites before being digested and ligated into the IR-pEG100 vectors in place of the Basta cassette.

The sqk1-1 allele was created using the egg-cell promoter/enhancer CRISPR-Cas9 system described by ${ }^{41}$. The two gRNA cassette was created using a four primer overlapping PCR (see Supplemental Table 1) to amplify the 20 nt gRNA : U6-26 terminator : U6-29 promoter 
: 20nt gRNA from the pCBC-DT1T2 plasmid ${ }^{42}$. This PCR product was cloned via Bsal Golden Gate reaction into a version of the pHEE401E plasmid containing an additional NapA promoter : dsRED : Nos terminator at the Mfel restriction site. The dsRED cassette was amplified out of the Traffic Lines ${ }^{43}$ (primers in Supplemental Table 1) and provides a method for negative selection of the Cas9 transgene. T1 transformants were selected on Hygromycin plates and screened for SQK1 promoter deletion via PCR (primers in Supplemental Table 1).

To create the dCas9 + SQK1-IR system, the pHEE401E plasmid was digested with Ncol and EcoRI to remove the active Cas9 cassette. The dCas9 sequence was amplified from the pDIRECT_21B plasmid ${ }^{44}$. The Rps5a promoter for dCas9 was synthesized by ThermoFisher and cloned into the pHEE401E backbone via In-Fusion reaction (Clontech). A gRNA cassette was created and inserted via Golden Gate reaction as described above using primers in Supplemental Table 1. dCas9 targets SQK1 at the sequence ATACTCAAAAATTAATAGA. The gRNA - dCas9 complex was amplified (primers in Supplemental Table 1) and In-Fusion cloned (Clontech) into a Sacl digested pMJS064 (described above) that contains the SQK1-IR. The Rbs terminator for dCas9 was amplified from pHEE401E and In-Fusion cloned into the pMJS064 + dCas9 plasmid following digestion with Avrll (resulting plasmid named pMJS082). The dCas9 construct without the SQK1-IR was created by removing the SQK1-IR from pMJS082 via restriction digest with Spel and Xhol.

\section{Expression Analysis}

RNA was extracted from three biological replicates using TRIzol Reagent (Thermo Fisher) and then DNase I treated and cleaned-up with the TURBO DNase DNA-free kit (Thermo Fisher) according to manufacturer's protocol. cDNA synthesis was performed using an oligo $\mathrm{d}(\mathrm{T})$ primer and Tetro Reverse Transcriptase (Bioline). We performed qRT-PCR using the primers in Supplemental Table 1 and SYBR green supermix. Relative expression was determined using the $2-\Delta \Delta \mathrm{Ct}$ method, comparing the gene of interest to a housekeeping control gene (AXS2 or ACTIN 2, see Supplemental Table 1). Mean and standard deviations of 3 biological replicates are calculated by Graphpad Prism and shown as bar graphs with error bars.

\section{Bisulfite Amplicon Sequencing and Analysis}

Genomic DNA was treated with RNase A, then cleaned and recovered by phenol chloroform isolation. $500 \mathrm{pg}-2 \mu \mathrm{g}$ DNA was treated with sodium bisulfite using the EZ DNA 
Methylation kit (Zymo Research). Amplicons for sequencing were generated by PCR using degenerate primers (see Supplemental Table 1) for each locus using My Taq HS mix (Bioline) and gel purification. For each bisulfite conversion reaction performed, we also performed PCR on a control unmethylated locus (At2G20610) in order to calculate the conversion rate (see Supplemental Figure 1 for example).

To generate amplicon libraries, purified amplicons were pooled in equimolar ratios. 100 ng of purified amplicon DNA in $30 \mu \mathrm{L}$ volume were used for library preparation with the Illumina Nextera DNA Flex kit. BSAS libraries from December 2019 to present were created using a modified Nextera Flex protocol (first described by ${ }^{45}$ ). Per index, $1 \mu \mathrm{L}$ Tagmentation (BLT) beads from the Nextera Flex kit was diluted with $19 \mu \mathrm{L}$ of ultrapure water and combined with 30 $\mu \mathrm{L}$ sample DNA and $50 \mu \mathrm{L}$ lab-made $2 \mathrm{X}$ tagmentation buffer $(20 \mathrm{mM}$ Tris, $20 \mathrm{mM} \mathrm{MgCl}, 50 \%$ DMF) before being tagmented at $55^{\circ} \mathrm{C}$ for 15 minutes. Tagmenation was stopped by adding 20 $\mu \mathrm{L} 0.2 \%$ SDS and incubating at $37^{\circ} \mathrm{C}$ for 15 minutes. Beads were washed three times with 100 $\mu \mathrm{L}$ lab-made tagmentation wash buffer (10\% PEG8000 and $0.25 \mathrm{M} \mathrm{NaCl}$ in TE buffer).

Tagmented DNA was amplified directly from the beads via 6 cycles of PCR amplification using the PrimeSTAR GXL Polymerase kit (Takara) and dual indexed adapters (Illumina) in $45 \mu \mathrm{L}$ reactions. Finally, libraries were purified using $81 \mu \mathrm{L}$ SPRIselect beads (Beckman Coulter) or Nextera Purification Beads (Illumina), washed twice in $200 \mu \mathrm{L} 80 \% \mathrm{EtOH}$ and eluted off beads in $32 \mu \mathrm{L}$ ultrapure water.

We sequenced the resulting libraries with $300 \mathrm{nt}$ single-end reads on the Illumina MiSeq platform at the University of Delaware DNA Sequencing and Genotyping Center. Raw reads were trimmed for adapters and mapped to all the amplicons pooled together for sequencing using methylpy (https://github.com/yupenghe/methylpy) ${ }^{46}$. Reference DNA sequences have primer sequences removed. The allC files (see Data and Code Availability) containing methylation data for each cytosine were used as input for Bedtools ${ }^{47}$ to calculate methylation percentage for each locus and plotted using ggplot2 in R. 95\% Wilson confidence intervals were calculated as in ${ }^{48}$ for error bars. BSAS data of DNA methylation levels was quantified per amplicon as the average methylation at each cytosine sequence context ( $\mathrm{CG}, \mathrm{CHG}, \mathrm{CHH})$. The total number of reads reporting the methylation status of a locus is noted as BSAS coverage. Data was analyzed in $\mathrm{R}$ and plotted with ggplot2.

\section{Bisulfite Sanger Sequencing and Analysis}

Amplicons for Sanger sequencing from Supplemental Figure 1 were generated as above for BSAS. Purified amplicons were subjected to single colony purification by TOPO TA cloning 
into pCR4 (Thermo Fisher) and transformation into E. coli. Individual colonies were sequenced by Sanger sequencing (Eton Biosciences) and analyzed in Kismeth ${ }^{49}$ using default parameters.

\section{Analysis of Whole Genome Bisulfite Sequencing Data}

Genome-wide MethylC-seq data is publicly available for wt Col Arabidopsis inflorescence ${ }^{50}$. Processed data was downloaded from GEO (GSM2101949). Similar to BSAS analysis, the allC file was used as input for Bedtools ${ }^{47}$ to calculate methylation percentage for each locus shown in Supplemental Figure 1.

\section{Determination of RdDM Strength}

To measure RdDM strength, we calculated the number of consecutive cytosines that are methylated for a given locus per sequencing read. Using methylpy ${ }^{46}$ we isolated the reads that mapped to a specific locus and used these as input for Kismeth ${ }^{49}$. The Kismeth output displays an image of methylation status of each individual cytosine along each individual read, and this was used for image analysis. We used a custom python script (https://github.com/jpeasari/DotPlot-Anaysis-OpenCV) to analyze each read in the image, represented by one row of methylation data and determined the longest stretch of consecutive methylated cytosines in each row. We summarized the longest stretch counts for each locus in box plots using ggplot2 in $R$.

\section{Small RNA Sequencing and Analysis}

Small RNA was sequenced as in ${ }^{17}$. Briefly, Trizol reagent (Thermo Fisher) was used to isolate total RNA. The mirVana miRNA isolation kit (Thermo Fisher) was used to enrich small RNAs. The TruSeq Small RNA Library Preparation kit (Illumina) was used to generate libraries and multiplexed for sequencing on a HiSeq 4000, HiSeq X or NextSeq 550 system at the University of Delaware DNA Sequencing and Genotyping Center or Novogene Inc.

Post-sequencing, the Illumina universal adapter was removed from the demultiplexed libraries using the fastx toolkit. Total genome matching reads were calculated using bowtie (parameters: - $v 0$ ), and this value is used for library depth normalization. The $s R N A$ Workbench ${ }^{51}$ was used to filter out t/rRNA reads, low complexity reads and retain only 18-28 nt small RNA reads that map the Arabidopsis Araport11 genome. To map the small RNAs to the genome, ShortStack ${ }^{52}$ was used with parameters: --nohp --mmap f --bowtie_m all --align_only. For assaying siRNAs from a specific transgene, bowtie (parameter: -v 0 --best --strata -M 1) was used to map the small RNAs to the full transgene sequence. Bedtools was used to count 
the number of reads mapping a specific locus. In Figure 3D, replicate 2 of ago4/6/9 contains the 35S:EVD transgene (from Figure 2B). The small RNAs mapping the transgene were removed before analysis for Figure 3D. In Figure 4A, de novo clusters of 23-24 nt siRNAs were called using ShortStack with a threshold of at least 10 raw reads in wt Col and Pol V-dependence was determined by at least a $\geq 2$-fold loss of siRNAs in pol $V$ mutants. Conversely, loci that did not lose siRNAs in pol V compared to wt Col were categorized as Pol V-independent siRNA loci. ggplot2 in R was used to generate siRNA heatmaps.

\section{AGO4-incorporated small RNA library preparation and analysis}

Frozen inflorescence tissue was ground with liquid nitrogen and resuspended in lysis buffer (50 mM Tris pH 8, 150 mM NaCl, 5 mM MgCl , 10\% glycerol, 1\% IGEPAL, 0.5 mM DTT, $1 \mathrm{mM}$ PMSF, $1 \mathrm{X}$ Roche protease inhibitor cocktail) and homogenized with mixing for 15 minutes at $4^{\circ} \mathrm{C}$. Lysates were then clarified with a spin. Clarified lysates were combined with $2 \mu \mathrm{L}$ of AGO4 antibody (Agrisera) or $2 \mathrm{uL}$ of rabbit IgG as a mock IP (Cell Signaling Technology), and rotated at $4^{\circ} \mathrm{C}$ for 1 hour. Immune complexes were harvested with $40 \mu \mathrm{L}$ of Protein $\mathrm{G}$ Dynabeads (Thermo), pre-washed in $1 \mathrm{X}$ TBS, rotating $30 \mathrm{~min}$ at $4^{\circ} \mathrm{C}$. Beads and immune complexes were washed $3 \mathrm{X}$ with $1 \mathrm{~mL}$ of cold wash buffer $(50 \mathrm{mM}$ Tris $\mathrm{pH} 8,150 \mathrm{mM} \mathrm{NaCl}, 5$ $\mathrm{mM} \mathrm{MgCl} 2,0.5 \mathrm{mM}$ DTT). Immunoprecipitated small RNA (bound by AGO4) was released from beads and isolated by TRIsure (Bioline) extraction. All RNA recovered from AGO4 IPs went directly into small RNA library preparation.

The small RNA library was prepared using TruSeq Small RNA Library Preparation kit (Illumina) as described above for total small RNAs with the exception of using 14 cycles of PCR amplification. Small RNAs were processed exactly as described earlier and accumulation was calculated in reads per million (RPM) sequenced reads for each size class of small RNAs in AGO4-IP and mock-IP samples. The small RNA enrichment was calculated as the ratio of RPM values for AGO4-IP over mock samples for each size class. This enrichment value is displayed as heatmap in Figure $2 \mathrm{C}$ and Supplemental Figure 6.

\section{Plasmid-Safe PCR Assay}

Frozen inflorescence tissue was ground with liquid nitrogen, and total DNA was purified using the QIAGEN DNeasy Plant Mini Kit. $1 \mathrm{ug}$ of resulting RNased DNA was digested using 1 ul Plasmid Safe ATP-dependent DNase (Lucigen) in a 50 ul reaction for 16 hours at $37^{\circ} \mathrm{C}$. Digestion was completed by twice adding $1 \mathrm{ul}$ additional DNase and $1 \mathrm{ul}$ additional ATP 
followed by two hours of incubation at $37^{\circ} \mathrm{C}$ for a total of 20 hours digestion. DNase was inactivated at $70^{\circ} \mathrm{C}$ for $30 \mathrm{~min}$. Digested and undigested DNA from each line was amplified using PCR primers for the EVD coding region (Supplemental Table 1).

\section{Western Blotting}

Frozen inflorescence tissue was ground with liquid nitrogen and resuspended in lysis buffer (50 mM Tris pH 8, 150 mM NaCl, 5 mM MgCl $2,10 \%$ glycerol, 1\% IGEPAL, 0.5 mM DTT, $1 \mathrm{mM}$ PMSF, $1 \mathrm{X}$ Roche protease inhibitor cocktail) and homogenized with mixing for 15 minutes at $4^{\circ} \mathrm{C}$. Lysates were clarified with a spin, combined with $2 \mathrm{X}$ loading buffer, denatured, and then loaded onto a 4\%-20\% gradient Tris-Glycine gel (Thermo). Protein was transferred from the gel to a PVDF membrane using the BioRad semi-dry transblot. Membranes were blocked for 1 hour at room temperature in 3\% milk powder $1 \mathrm{X}$ PBS-T. Primary antibodies, which include Pol V (Wierzbicki laboratory), AGO4 (Agrisera), and ACT11 (Agrisera), were all diluted 1:1000 in 3\% milk 1X PBS-T solution and incubated on blots overnight. Washes were performed at room temperature with $1 \mathrm{X}$ PBS-T. Anti-rabbit secondary antibody (Sigma) was used for visualization of Pol V and AGO4, while anti-mouse secondary (Sigma) was used for ACT11. Blots were visualized using HRP chemiluminescence (Thermo), with exposures ranging from 5 seconds to 5 minutes.

\section{Chromatin IP and Quantitative PCR}

Nuclei were crosslinked as follows: frozen inflorescences (300 mg per biorep) were ground with liquid nitrogen and resuspend in nuclear isolation buffer (10 mM HEPES, $1 \mathrm{M}$ sucrose, $5 \mathrm{mM} \mathrm{KCl}, 5 \mathrm{mM} \mathrm{MgCl}$, 0.6\% Triton X-100, $0.4 \mathrm{mM} \mathrm{PMSF,} 1$ X Roche protease inhibitor cocktail) and homogenized with mixing for 15 minutes at $4^{\circ} \mathrm{C}$. Methanol-free formaldehyde (Pierce) was added to a final concentration of $1 \%$ with end-over-end mixing for 15 minutes at room temperature. Formaldehyde crosslinking was quenched with glycine (125 $\mathrm{mM})$, rotating 5 minutes at room temperature. Crosslinked nuclei were then filtered through two layers of Miracloth to remove large particles before centrifugation at $3000 \mathrm{xg}$ for 15 minutes at $4^{\circ} \mathrm{C}$. The resulting nuclear pellet was resuspended in wash buffer $(10 \mathrm{mM}$ Tris $\mathrm{pH} 8,0.25 \mathrm{M}$ sucrose, $10 \mathrm{mM} \mathrm{MgCl}_{2}, 1 \mathrm{mM}$ EDTA, 1\% Triton X-100, 1X Roche protease inhibitor cocktail) and nuclei were cleaned and pelleted at $12,000 \times \mathrm{g}$ for $10 \mathrm{~min}$ at $4^{\circ} \mathrm{C}$. The final clean nuclear pellet was resuspended in $1 \mathrm{~mL}$ nuclear lysis buffer (20 mM Tris $\mathrm{pH} 8,2 \mathrm{mM}$ EDTA, 0.1\% SDS, $1 \mathrm{mM}$ PMSF, 1X Roche protease inhibitor cocktail) and sonicated on the Covaris E220 (150W peak power, $20 \%$ duty factor, 200 cycles/burst, 6 minutes). Insoluble debris was removed from the 
sonicated soluble chromatin by centrifugation at $12,000 \times \mathrm{g}$ for 10 minutes at $4^{\circ} \mathrm{C}$. $30 \mu \mathrm{L}$ of $5 \mathrm{M}$ $\mathrm{NaCl}$ and $20 \mu \mathrm{L}$ of $30 \%$ Triton $\mathrm{X}-100$ were both added to $920 \mu \mathrm{L}$ of sonicated chromatin in order to sequester SDS before immunoprecipitation. 3\% input by volume was set aside for each sample, and the remaining volume of sonicated chromatin was divided evenly among IPs and IgG negative controls for overnight immunoprecipitation with respective antibodies or lgG.

Pol V (Lagrange lab antibody) and Ser5P Pol II (Abcam) ChIPs were performed with 2 $\mu \mathrm{L}$ antibody, whereas AGO4 (Agrisera) and H3K9me2 (Abcam) ChIPs used $5 \mu \mathrm{L}$ antibody per IP. Each experiment used the same volume of rabbit IgG as a negative control (Cell Signaling Technology). Immune complexes were collected using $40 \mu \mathrm{L}$ of washed protein A/G magnetic beads (Pierce), rotating at $4^{\circ} \mathrm{C}$ for 2 hours. After collecting immune complexes, beads were washed at $4^{\circ} \mathrm{C}$ using one rinse and two 5-minute washes of each of the following buffers: low salt (150 mM NaCl, 0.1\% SDS, 1\% Triton X-100, 2 mM EDTA, $20 \mathrm{mM}$ Tris pH 8), high salt (500 $\mathrm{mM} \mathrm{NaCl}, 0.1 \%$ SDS, 1\% Triton X-100, 2 mM EDTA, 20 mM Tris pH 8), LiCl buffer (250 mM $\mathrm{LiCl}, 1 \%$ Igepal, $1 \%$ sodium deoxycholate, $1 \mathrm{mM}$ EDTA, $10 \mathrm{mM}$ Tris $\mathrm{pH} 8$ ), and TE $+0.1 \%$ Igepal. Chromatin was eluted from the beads using $250 \mu \mathrm{L}$ elution buffer ( $1 \%$ SDS, $0.1 \mathrm{M}$ $\mathrm{NaHCO}_{3}$ ) at $65^{\circ} \mathrm{C}$ for 15 minutes with agitation. Overnight reverse crosslinking was accomplished with the addition of $20 \mu \mathrm{L} 5 \mathrm{M} \mathrm{NaCl}$ at $65^{\circ} \mathrm{C}$ for all samples including inputs. Proteinase $\mathrm{K}$ digestion was performed by the addition of $10 \mu \mathrm{L} 0.5 \mathrm{M}$ EDTA, $20 \mu \mathrm{L} 1 \mathrm{M}$ Tris pH 7, 20 ug Proteinase K (Thermo Scientific) and $50 \mathrm{ng}$ RNase A, incubating at $42^{\circ} \mathrm{C}$ for one hour. DNA was then purified using DNA Clean and Concentrator-25 columns (Zymo Research) and eluted in $100 \mu \mathrm{L}$ of elution buffer.

Quantitative PCR was performed using primers in Supplemental Table 1 and Sso Universal SYBR (BioRad). Percent input was calculated by first normalizing the Ct values of the diluted input samples to $100 \%$ input by the following calculation: $\mathrm{Ct}_{100 \%}=\mathrm{Ct}_{\text {diluted }}$ - $(\log$ (dilution factor, 2)). Then the IgG and IP Ct values were normalized to this new $100 \%$ input Ct value using the $2^{-\Delta \Delta C t}$ method, which represents \% input. For Pol V and AGO4 ChIP, the pol V mutant and ago4/6/9 mutants (ago4-4 allele, a complete null) were respectively used to calculate background levels for each of these antibodies. These background levels were averaged for each PCR target. They were then subtracted from the \% input values of each of the other genotypes. Mean and standard deviations of the biological replicates are shown as bar graphs with error bars.

\section{ChIP-seq and RIP-seq Data Analysis}




\section{Article}

Raw reads were downloaded from NCBI GEO (GSE52041 and GSE70290), trimmed for adapters and mapped to the Araport11 Arabidopsis genome. The ChIP-seq reads were mapped using Shortstack (parameters: --nohp --mmap f --bowtie_m all). The RIP-seq reads were mapped using Soapsplice $1.10^{53}$ using parameters: -t 10300 (maximum distance between two segments) as mentioned in the original study ${ }^{9}$. For Figure $3 \mathrm{~A}$, reads were counted using Bedtools for each individual locus. To determine enrichment, the normalized ratio of IP over mock sample reads was calculated (for both ChIP-seq and RIP-seq). In eukaryotes, organelleto-nucleus DNA transfer is known to occur which has resulted in some mitochondrial genes being duplicated and part of the nuclear genome. To avoid such genes causing an artefact of Pol V enrichment in the negative control dataset, we analyzed only those mitochondrial genes which do not have any similarity to nuclear genes.

In Figure 3C, genome-wide Pol V enriched loci were determined using the Macs2 ChIP peak caller ${ }^{54}$. Pol $\mathrm{V}$ fold enrichment was calculated using the ratio of IP over mock samples. The two replicates were averaged for wt Col and suvh2/suvh9 mutant. The loci that had $\geq 2$-fold Pol

V enrichment in wt Col and only background levels of Pol V enrichment in suvh2/suvh9 were categorized as SUVH2/SUVH9-fully dependent Pol V loci. The loci that retained at least $\geq 2$-fold Pol V enrichment in suvh2/suvh9 mutants were categorized as SUVH2/SUVH9-partially dependent Pol V loci. In Figure 4C, AGO4 enrichment was calculated as a ratio of IP over mock normalized read accumulation. For genome-wide analyses, the two biological replicates were averaged, whereas the replicates are also displayed individually in Figure 3A.

\section{Data Availability}

Raw Illumina sequencing data produced for this study is available from $\mathrm{NCBI}$ as GSEXXXXXX. Additional small RNA datasets were downloaded from GSE118705. Processed BSAS data of DNA methylation levels is available as Supplemental Dataset 1. Sanger sequencing results are available as Supplemental Dataset 2.

\section{Code Availability}

The custom script used to generate RdDM Strength plots is available on Github https://github.com/jpeasari/Dot-Plot-Anaysis-OpenCV.

\section{Material Availability}




\section{Article}

Biological materials can be obtained from the corresponding author without restriction.

\section{Acknowledgements}

The authors thank Diego Cuerda-Gil, Seth Edwards, and Peng Liu for generating small RNA sequencing libraries. We thank Thierry Lagrange, Craig Pikaard and Andrzej Wierzbicki for sharing NRPE1 seed and antibody resources, Mike Axtell for ago4-4/6/9 seed, and Yijun Qi for $d c / 1 / 2 / 3 / 4$ seed. We thank Jennifer Mele at The Ohio State Genomics Shared Resource, the University of Delaware DNA Sequencing \& Genotyping Center and the Donald Danforth Plant Science Center Data Science Facility for computing support. We also thank the Donald Danforth Plant Science Center Plant Growth Facility, Gary Posey at the Center for Applied Plant Sciences Greenhouse, and Emily Yoders-Horn and David Snodgrass at the Biological Sciences Greenhouse. This work is supported by NSF grant MCB-1908521 to R.K.S.

\section{Author Contributions}

Conceptualization - M.J.S., K.P., R.K.S., A.D.M.; Methodology - M.J.S., K.P., R.K.S., A.D.M.; Software - J.P., K.P.; Investigation - M.J.S., R.K., L.L.M., H.P., K.P., A.D.M.; Resources A.Y.H.; Data Curation - K.P.; Writing - M.J.S., K.P., R.K.S. and A.D.M.; Funding Acquisition R.K.S.

\section{Competing Interests Statement}

The authors declare no competing interests.

\section{Figure Legends}

Figure 1 - SUVH2/SUVH9-independent function of Pol V

A. Bisulfite Amplicon Sequencing (BSAS) of the 35S:EVD transgene in independent biological replicates of $\mathrm{T} 1$ plants. Error bars represent $95 \%$ Wilson confidence intervals. $\mathrm{H}=\mathrm{A}, \mathrm{C}$ or $\mathrm{T}$.

B. RdDM strength plot of DNA methylation. RdDM strength was measured by calculating the length of stretches of consecutively methylated cytosines along individual sequence reads. Box plots represent 25th and 75th percentile values with whiskers at the 10 th and 90th percentile, the median is represented by a line, and the mean is denoted by a filled circle.

\section{Figure 2 - Genetic requirements for the first round of RdDM}


A. BSAS of 35S:EVD in T1 plants lacking key RdDM factors demonstrates that the initiation of DNA methylation is dependent upon Pol V, DRM1/2, and at least one member of the AGO4-clade of proteins.

B. Heatmap of small RNA sequencing for transgenic lines from part $A$. The accumulation of different sized siRNAs is shown on the Y-axis, with the key sizes 21, 22 and $24 \mathrm{nt}$ in red. RPM = reads per million genome-mapped sequences. The siRNAs shown were mapped to the entire length of the EVD coding region, not just the region analyzed in part $A$ with BSAS.

C. Heatmap of EVD small RNA enrichment in AGO4. Enrichment is calculated as the ratio of small RNA accumulation (RPM) in AGO4-IP over mock-IP samples for each size class of small RNA.

D. ChIP analysis of Pol II (Ser5P) in T1 transgenic plants from part A shown as \% input. Error bars represent the standard deviation. Three biological replicates were used for each genotype. SimpleHat2 is a transcriptionally silenced TE negative control, and AXS2 is a transcribed positive control gene.

E. ChIP analysis of H3K9me2 levels in $\mathrm{T} 1$ transgenic plants shown as \% input. Three biological replicates were used for each genotype. Athila6 is a silenced TE with known accumulation of $\mathrm{H} 3 \mathrm{~K} 9 \mathrm{me} 2$.

F. BSAS of 35S:EVD in T1 plants lacking various combinations of DCL family proteins. The $d c / 1 / 2 / 3 / 4$ quadruple mutant plants are siblings of the $d c / 2 / 3 / 4 ; d c / 1 /+$ plants (siblings denoted with *).

G. Heatmap of small RNA sequencing for the same transgenic lines as in part F.

\section{Figure 3 - The first round of Pol V recruitment requires an AGO4-clade protein}

A. Reanalysis of ChIP and RIP data of Pol V occupancy and transcription. Mitochondrial negative control genes, nuclear genes and nuclear positive control RdDM regions are shown. Two replicates are shown as dots, and their average is the height of the bar. N/A $=$ Not applicable due to absence of any mapping reads in the IP or the mock samples. Genes with colored labels are used as controls in ChIP experiments.

B. Pol V ChIP of 35S:EVD T1 plants as \% input minus the background of \% input in T1 pol $V$ mutants. At least three biological replicates were used for each genotype. At5G52070 is a locus that undergoes RdDM and serves as a positive control for Pol V occupancy ${ }^{32}$, while AXS2 is a gene that does not undergo RdDM and serves as a negative control. 
Error bars represent the standard deviation. Statistical significance was determined by a two-tailed unpaired t-test with Welch's correction.

C. Box plot with connected data points of Pol V occupancy comparing wt Col (blue) and suvh2/suvh9 double mutants (yellow). Pol V occupied regions are categorized into SUVH2/SUVH9 partially-dependent and fully-dependent loci (see Methods). Pol V occupancy is displayed as fold enrichment over mock-IP. Grey lines represent the change in Pol V enrichment between wt Col and suvh2/suvh9 for each locus. Box plots represent 25 th and 75 th percentile with whiskers at 10 th and 90th percentile values, and the median is represented by a line.

D. Heatmap of small RNA sequencing for Pol V occupied loci that are categorized as either SUVH2/SUVH9 partially-dependent or fully-dependent from part C.

\section{Figure 4 - AGO4 can localize to chromatin loci independent of Pol V}

A. Box plot with connected data points of 23-24 nt siRNA accumulation in wt Col (blue) and pol $V$ mutants (yellow). SiRNA loci are defined as Pol V-dependent or -independent based on change in siRNA accumulation in pol $V$ compared to wt Col. Box plot percentiles are the same as in Figure 3C.

B. Venn diagram showing the overlap of Pol V-independent siRNA loci from part $A$ and AGO4-enriched loci as previously defined ${ }^{27}$. The overlap provides 91 testable loci for part C.

C. Box plot with connected data points of AGO4 protein enrichment. AGO4 occupancy is displayed as fold enrichment over mock-IP. Grey lines display loci that lose AGO4 occupancy in pol $V$ mutants (31\%). Red lines display loci that retain AGO4 occupancy in the pol $V$ mutant background (69\%).

D. AGO4 ChIP of 35S:EVD T1 plants as \% input minus the background of \% input in T1 ago4/6/9 mutants. Bar graph display is the same as in Figure 3B. At5G52070 is a positive control locus that undergoes RdDM, and AXS2 is a negative control gene that does not go through RdDM.

E. AGO4 ChIP of 35S:EVD T1 plants as \% input minus the background of \% input in T1 ago4/6/9 mutant plants. Bar graph display is the same as in Figure 4D.

\section{Figure 5 - siRNAs are sufficient to target the first round of RdDM}

A. Two component system with siRNA production from a T1 inverted repeat (IR) transgene targeting the endogenous gene SQK1 for RdDM. 
B. Heatmap of small RNA sequencing.

C. BSAS for T1 transgenic SQK1-IR plants.

D. Box plots of RdDM strength for plants from C.

\section{Figure 6 - Pol II's role in the first round of RdDM}

A. CHH context DNA methylation and siRNAs (combined 21, 22 and $24 \mathrm{nt}$ ) aligned to the endogenous SQK1 gene. The region of SQK1 that matches the SQK1-IR transgene is annotated. The track below the SQK1-IR of wt $\mathrm{Col} \mathrm{CHH}$ methylation identifies where each $\mathrm{CHH}$ context cytosine is located (green), even if unmethylated. The DNA methylation track for SQK1-IR in wt Col is produced from BSAS of two overlapping amplicons. Dashed lines align key annotation features of the SQK1 gene, and the location of the dCas 9 gRNA sequence is annotated.

B. qRT-PCR of plants homozygous for the SQK1 promoter deletion allele sqk1-1. The error bar represents the standard deviation of three biological replicates.

C. BSAS of plants with and without the SQK1-IR, the homozygous sqk1-1 deletion and dCas9.

D. Depiction of three EVD TE transgenes. These transgenes share the exact coding sequence of EVD5 (AT5TE20395) lacking any vector-encoded terminator sequence while differing in their upstream elements. These upstream elements include the $35 \mathrm{~S}$ constitutive promoter (from Figure 1), no promoter and the T3A terminator.

E. qRT-PCR of EVD mRNA in T1 transgenic plants indicates that only 35S:EVD has Pol II expression.

F. BSAS of T1 transformants for each respective EVD transgene in wt Col.

G. Heatmap of small RNA sequencing from single and double EVD transgenic plants. The siRNAs shown were mapped to the entire length of the EVD coding region, not just the region analyzed by BSAS in part $F$.

H. BSAS for the 35S:EVD transgene (top) and the second unexpressed EVD transgene (bottom) in the same transgenic individuals. N/A = not applicable, as that transgene is not in this plant line. Biological replicates are shown in Supplemental Figure 8.

\section{Figure 7 - Model of RNA-driven recruitment of Pol V to new RdDM targets}

The initiation of RdDM begins with Pol II expression of the unmethylated locus (step 1), followed by siRNA production from that transcript by any member of the DCL family (step 2). SiRNAs are incorporated into a member of the AGO4-clade of proteins (step 3) and this can only target 
RdDM to complementary regions of the genome that are expressed (step 4). Cis action refers to a region that generates siRNAs and targets its own methylation, while in trans action the siRNAs are acting on a separate locus. The AGO4 targeting of a Pol II expressed region of the genome results in Pol V recruitment (step 5) and the first round of RdDM (step 6). This final methylated product can then enter the methylation-dependent pathway of continual Pol V recruitment guided by SUVH2/SUVH9. See Discussion section for details. Created with BioRender.com.

\section{References}

1. Deniz, Ö., Frost, J. M. \& Branco, M. R. Regulation of transposable elements by DNA modifications. Nat Rev Genet 20, 417-431 (2019).

2. Morel, J.-B., Mourrain, P., Béclin, C. \& Vaucheret, H. DNA methylation and chromatin structure affect transcriptional and post-transcriptional transgene silencing in Arabidopsis. Curr Biol 10, 1591-1594 (2000).

3. Hamilton, A., Voinnet, O., Chappell, L. \& Baulcombe, D. Two classes of short interfering RNA in RNA silencing. EMBO J 21, 4671-4679 (2002).

4. Thomas, A. L. et al. Piwi induces piRNA-guided transcriptional silencing and establishment of a repressive chromatin state. Gene Dev 27, 390-399 (2013).

5. Zhang, H., Lang, Z. \& Zhu, J.-K. Dynamics and function of DNA methylation in plants. Nat Rev Mol Cell Bio 19, 489-506 (2018).

6. Havecker, E. R. et al. The Arabidopsis RNA-Directed DNA Methylation Argonautes Functionally Diverge Based on Their Expression and Interaction with Target Loci. Plant Cell 22, 321-334 (2010).

7. Cao, X. et al. Role of the DRM and CMT3 Methyltransferases in RNA-Directed DNA Methylation. Curr Biol 13, 2212-2217 (2003).

8. Wierzbicki, A. T., Haag, J. R. \& Pikaard, C. S. Noncoding Transcription by RNA Polymerase Pol IVb/Pol V Mediates Transcriptional Silencing of Overlapping and Adjacent Genes. Cell 135, 635-648 (2008).

9. Böhmdorfer, G. et al. Long non-coding RNA produced by RNA polymerase $V$ determines boundaries of heterochromatin. Elife 5, e19092 (2016).

10. Ream, T. S. et al. Subunit Compositions of the RNA-Silencing Enzymes Pol IV and Pol V Reveal Their Origins as Specialized Forms of RNA Polymerase II. Mol Cell 33, 192-203 (2009). 11. Liu, Z.-W. et al. The SET Domain Proteins SUVH2 and SUVH9 Are Required for Pol V Occupancy at RNA-Directed DNA Methylation Loci. Plos Genet 10, e1003948 (2014).

12. Johnson, L. M. et al. SRA- and SET-domain-containing proteins link RNA polymerase $V$ occupancy to DNA methylation. Nature 507, 124-128 (2014).

13. Zheng, B. et al. Intergenic transcription by RNA Polymerase II coordinates Pol IV and Pol V in siRNA-directed transcriptional gene silencing in Arabidopsis. Gene Dev 23, 2850-2860 (2009).

14. Li, S. et al. Detection of Pol IV/RDR2-dependent transcripts at the genomic scale in 
Arabidopsis reveals features and regulation of siRNA biogenesis. Genome Res 25, 235-245 (2015).

15. Tsuzuki, M. et al. Broad noncoding transcription suggests genome surveillance by RNA polymerase V. Proc National Acad Sci 117, 30799-30804 (2020).

16. Bond, D. M. \& Baulcombe, D. C. Epigenetic transitions leading to heritable, RNA-mediated de novo silencing in Arabidopsis thaliana. Proc National Acad Sci 112, 917-922 (2015).

17. Nuthikattu, S. et al. The initiation of epigenetic silencing of active transposable elements is triggered by RDR6 and 21-22 nucleotide small interfering RNAs. Plant Physiol 162, 116-31 (2013).

18. Fultz, D. \& Slotkin, R. K. Exogenous Transposable Elements Circumvent Identity-Based Silencing, Permitting the Dissection of Expression-Dependent Silencing. Plant Cell 29, 360-376 (2017).

19. Marí-Ordóñez, A. et al. Reconstructing de novo silencing of an active plant retrotransposon. Nat Genet 45, 1029-1039 (2013).

20. Masser, D. R., Berg, A. S. \& Freeman, W. M. Focused, high accuracy 5-methylcytosine quantitation with base resolution by benchtop next-generation sequencing. Epigenet Chromatin 6, 33 (2013).

21. Pontier, D. et al. NERD, a Plant-Specific GW Protein, Defines an Additional RNAi-

Dependent Chromatin-Based Pathway in Arabidopsis. Mol Cell 48, 121-132 (2012).

22. Xie, Z. et al. Genetic and Functional Diversification of Small RNA Pathways in Plants. PLoS Biol 2, e104 (2004).

23. Onodera, Y. et al. Plant Nuclear RNA Polymerase IV Mediates siRNA and DNA MethylationDependent Heterochromatin Formation. Cell 120, 613-622 (2005).

24. Gao, Z. et al. An RNA polymerase II- and AGO4-associated protein acts in RNA-directed DNA methylation. Nature 465, 106-109 (2010).

25. Wierzbicki, A. T., Ream, T. S., Haag, J. R. \& Pikaard, C. S. RNA polymerase V transcription guides ARGONAUTE4 to chromatin. Nat Genet 41, 630-634 (2009).

26. Qi, Y. et al. Distinct catalytic and non-catalytic roles of ARGONAUTE4 in RNA-directed DNA methylation. Nature 443, 1008-1012 (2006).

27. Zheng, $Q$. et al. RNA polymerase $V$ targets transcriptional silencing components to promoters of protein-coding genes. Plant J 73, 179-189 (2013).

28. Béclin, C., Boutet, S., Waterhouse, P. \& Vaucheret, H. A Branched Pathway for TransgeneInduced RNA Silencing in Plants. Curr Biol 12, 684-688 (2002).

29. Zilberman, D. et al. Role of Arabidopsis ARGONAUTE4 in RNA-Directed DNA Methylation

Triggered by Inverted Repeats. Curr Biol 14, 1214-1220 (2004).

30. Sasaki, T. et al. Distinct and concurrent pathways of Pol II- and Pol IV-dependent siRNA biogenesis at a repetitive trans-silencer locus in Arabidopsis thaliana. Plant J 79, 127-138 (2014).

31. Butaye, K. M. J. et al. Stable high-level transgene expression in Arabidopsis thaliana using gene silencing mutants and matrix attachment regions. Plant J 39, 440-449 (2004).

32. Lahmy, S. et al. Evidence for ARGONAUTE4-DNA interactions in RNA-directed DNA methylation in plants. Gene Dev 30, 2565-2570 (2016).

33. Pikaard, C. S., Haag, J. R., Pontes, O. M. F., Blevins, T. \& Cocklin, R. A Transcription Fork Model for Pol IV and Pol V-Dependent RNA-Directed DNA Methylation. Cold Spring Harb Sym 
77, 205-212 (2012).

34. Sternberg, S. H., Redding, S., Jinek, M., Greene, E. C. \& Doudna, J. A. DNA interrogation by the CRISPR RNA-guided endonuclease Cas9. Nature 507, 62-67 (2014).

35. Matzke, M. A. \& Mosher, R. A. RNA-directed DNA methylation: an epigenetic pathway of increasing complexity. Nat Rev Genet 15, 394-408 (2014).

36. Baduel, P. et al. Genetic and environmental modulation of transposition shapes the evolutionary potential of Arabidopsis thaliana. Genome Biol 22, 138 (2021).

37. Papikian, A., Liu, W., Gallego-Bartolomé, J. \& Jacobsen, S. E. Site-specific manipulation of Arabidopsis loci using CRISPR-Cas9 SunTag systems. Nat Commun 10, 729 (2019).

38. Lister, R. et al. Highly Integrated Single-Base Resolution Maps of the Epigenome in Arabidopsis. Cell 133, 523-536 (2008).

39. Rowley, M. J., Avrutsky, M. I., Sifuentes, C. J., Pereira, L. \& Wierzbicki, A. T. Independent Chromatin Binding of ARGONAUTE4 and SPT5L/KTF1 Mediates Transcriptional Gene Silencing. PLoS Genet 7, e1002120 (2011).

40. Quintero-Cadena, P., Lenstra, T. L. \& Sternberg, P. W. RNA Pol II Length and Disorder Enable Cooperative Scaling of Transcriptional Bursting. Mol Cell 79, 207-220.e8 (2020). 41. Wang, Z.-P. et al. Egg cell-specific promoter-controlled CRISPR/Cas9 efficiently generates homozygous mutants for multiple target genes in Arabidopsis in a single generation. Genome Biol 16, 144 (2015).

42. Xing, H.-L. et al. A CRISPR/Cas9 toolkit for multiplex genome editing in plants. Bmc Plant Biol 14, 327 (2014).

43. Wu, G., Rossidivito, G., Hu, T., Berlyand, Y. \& Poethig, R. S. Traffic Lines: New Tools for Genetic Analysis in Arabidopsis thaliana. Genetics 200, 35-45 (2015).

44. Čermák, T. et al. A Multipurpose Toolkit to Enable Advanced Genome Engineering in Plants. Plant Cell 29, 1196-1217 (2017).

45. Gaio, D. et al. Hackflex: low cost Illumina sequencing library construction for high sample counts. Biorxiv 779215 (2019) doi:10.1101/779215.

46. Schultz, M. D. et al. Human body epigenome maps reveal noncanonical DNA methylation variation. Nature 523, 212-216 (2015).

47. Quinlan, A. R. \& Hall, I. M. BEDTools: a flexible suite of utilities for comparing genomic features. Bioinformatics 26, 841-842 (2010).

48. Henderson, I. R. et al. The De Novo Cytosine Methyltransferase DRM2 Requires Intact UBA Domains and a Catalytically Mutated Paralog DRM3 during RNA-Directed DNA Methylation in Arabidopsis thaliana. PLoS Genet 6, e1001182 (2010).

49. Gruntman, E. et al. Kismeth: Analyzer of plant methylation states through bisulfite sequencing. Bmc Bioinformatics 9, 371 (2008).

50. Panda, K. et al. Full-length autonomous transposable elements are preferentially targeted by expression-dependent forms of RNA-directed DNA methylation. Genome Biol 17, 170 (2016).

51. Stocks, M. B. et al. The UEA sRNA Workbench (version 4.4): a comprehensive suite of tools for analyzing miRNAs and sRNAs. Bioinformatics 34, 3382-3384 (2018).

52. Johnson, N. R., Yeoh, J. M., Coruh, C. \& Axtell, M. J. Improved Placement of Multi-mapping Small RNAs. G3 Genes Genomes Genetics 6, 2103-2111 (2016).

53. Huang, S. et al. SOAPsplice: Genome-Wide ab initio Detection of Splice Junctions from 


\section{Article}

RNA-Seq Data. Frontiers Genetics 2, 46 (2011).

54. Gaspar, J. M. Improved peak-calling with MACS2. Biorxiv 496521 (2018) doi:10.1101/496521.

55. Lanciano, S. et al. Sequencing the extrachromosomal circular mobilome reveals retrotransposon activity in plants. PLoS Genet 13, e1006630 (2017).

56. Tsukahara, S. et al. Bursts of retrotransposition reproduced in Arabidopsis. Nature 461, 423-426 (2009).

57. Menand, B. et al. An Ancient Mechanism Controls the Development of Cells with a Rooting Function in Land Plants. Science 316, 1477-1480 (2007).

58. Sullivan, A. et al. An 'eFP-Seq Browser' for visualizing and exploring RNA sequencing data. Plant J 100, 641-654 (2019). 


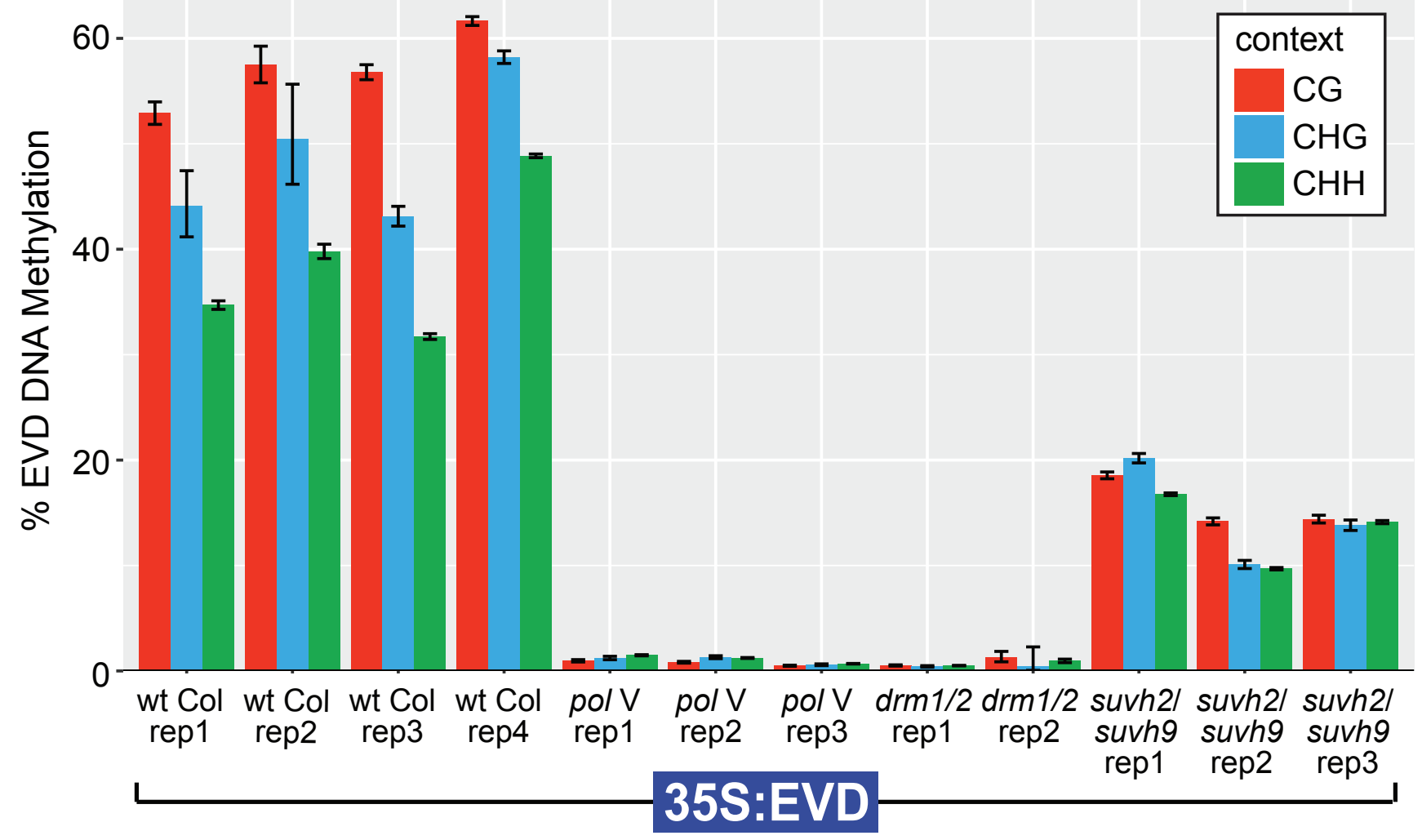

b

RdDM strength

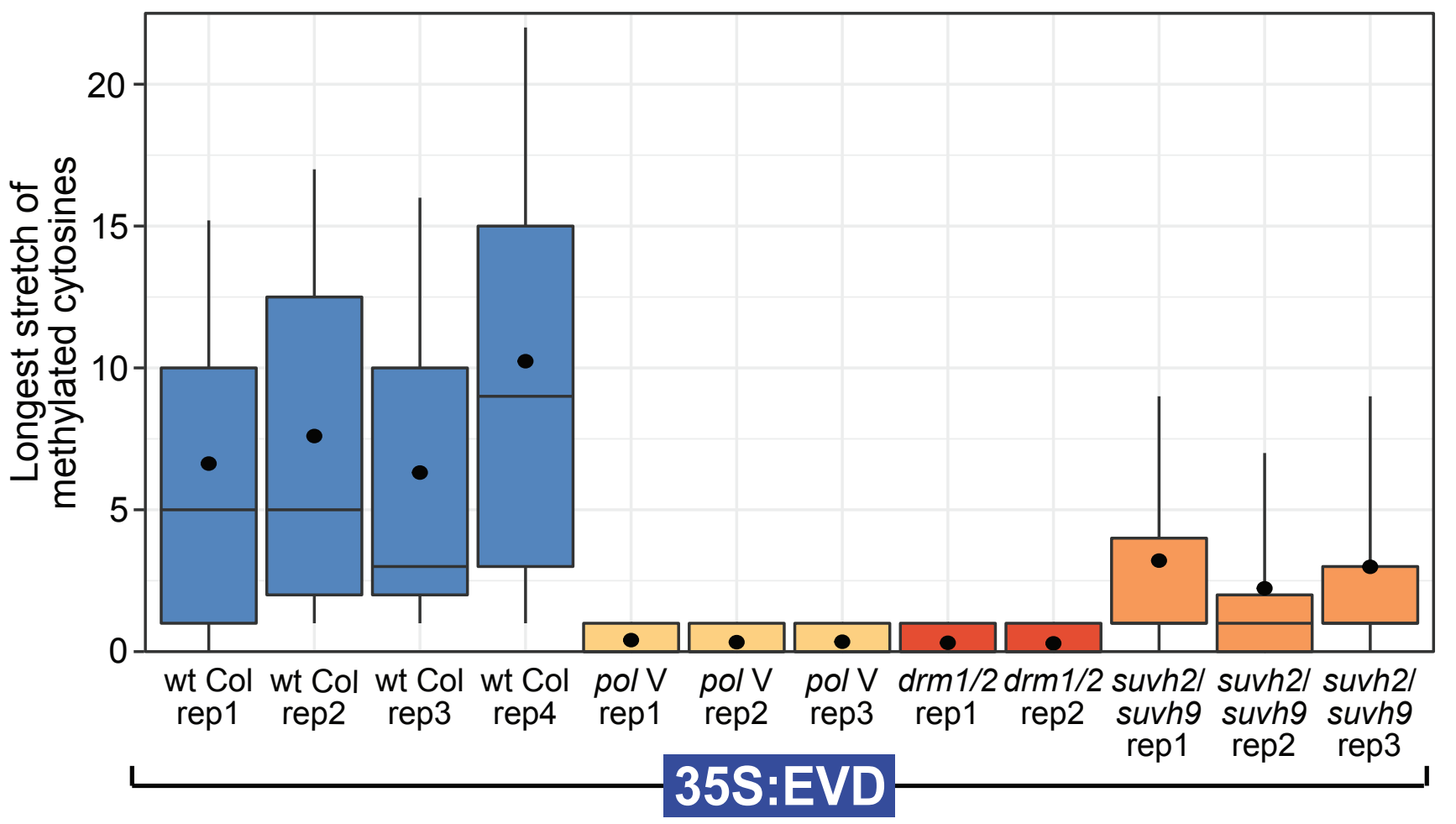


$\mathbf{a}$

DNA methylation in mutants of downstream RdDM factors

b

Small RNA-seq in mutants

of downstream RdDM factors

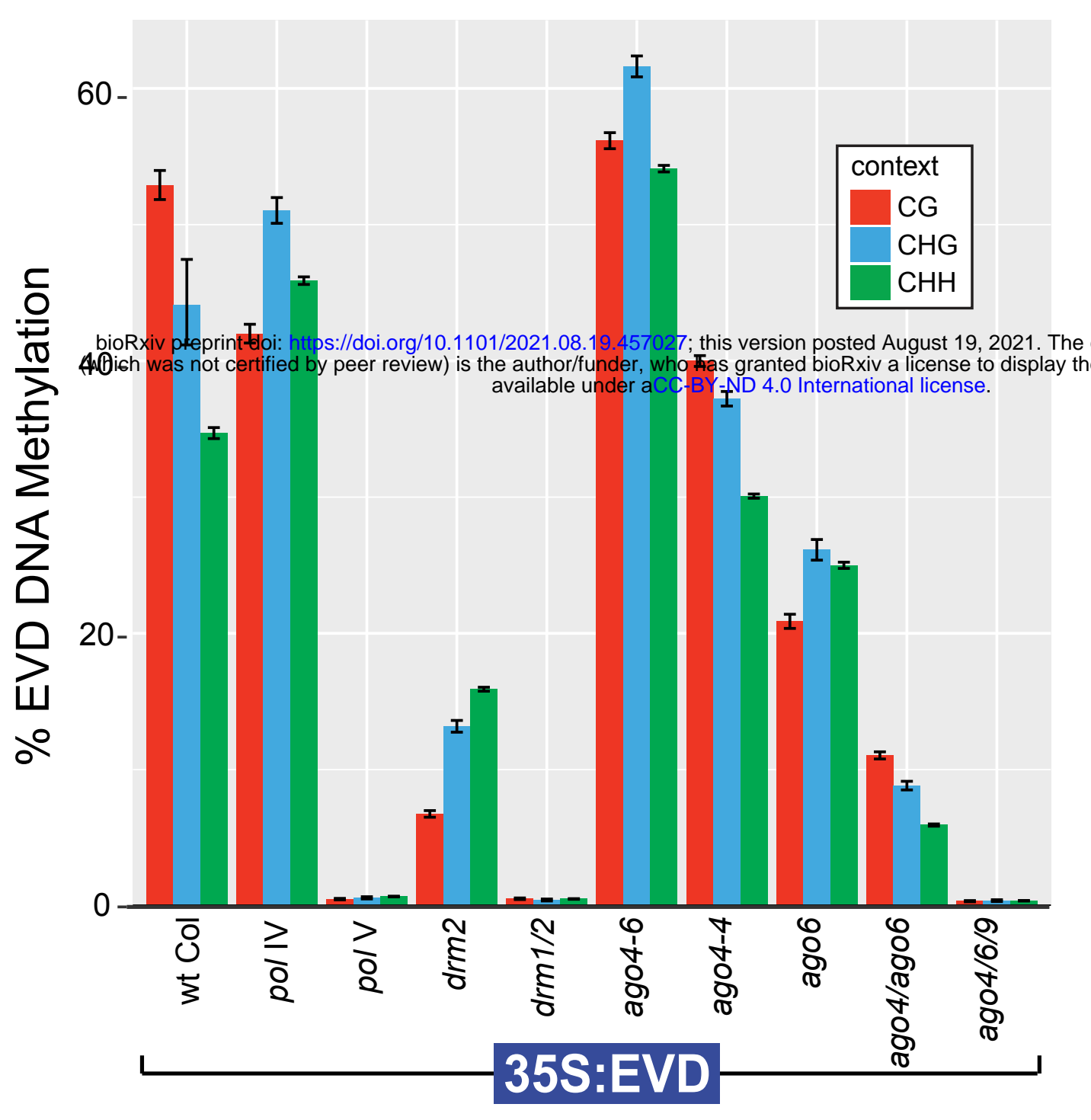

d

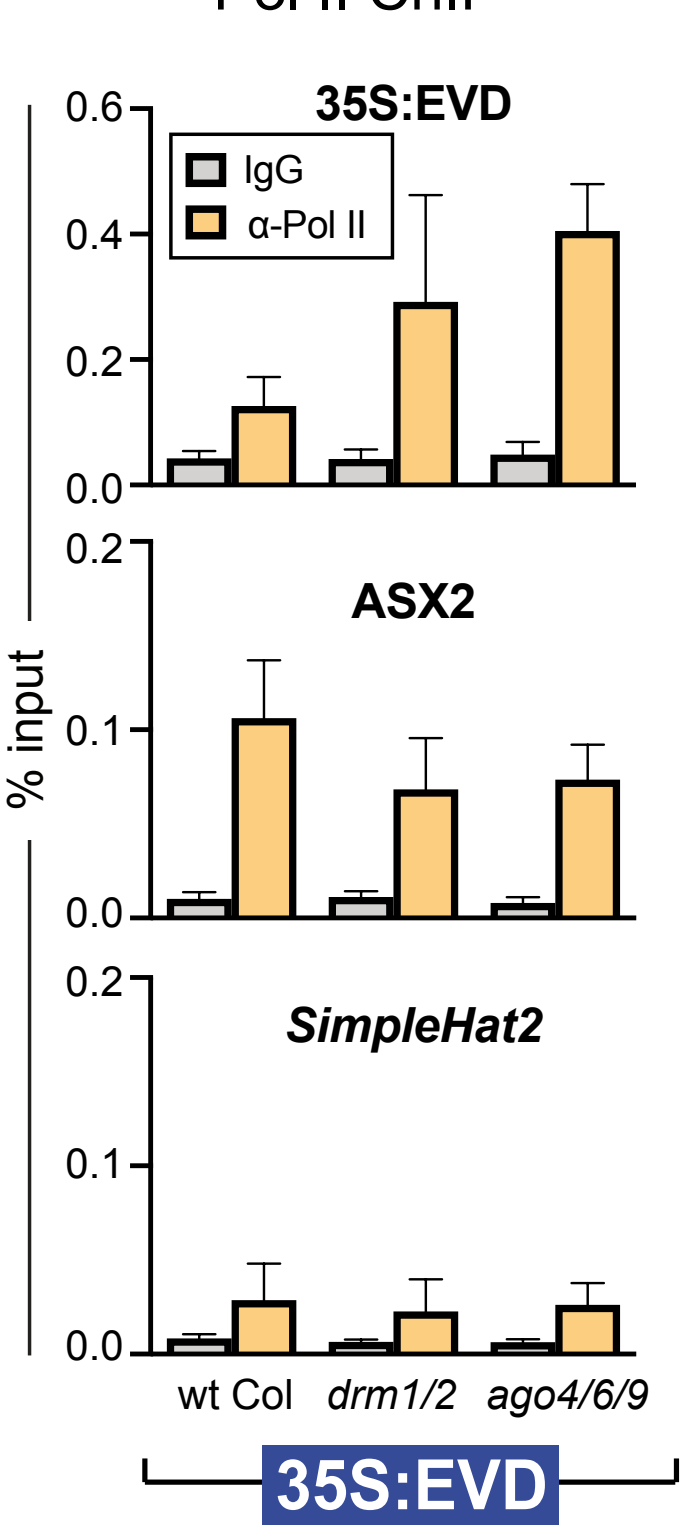

e H3K9me2 ChIP

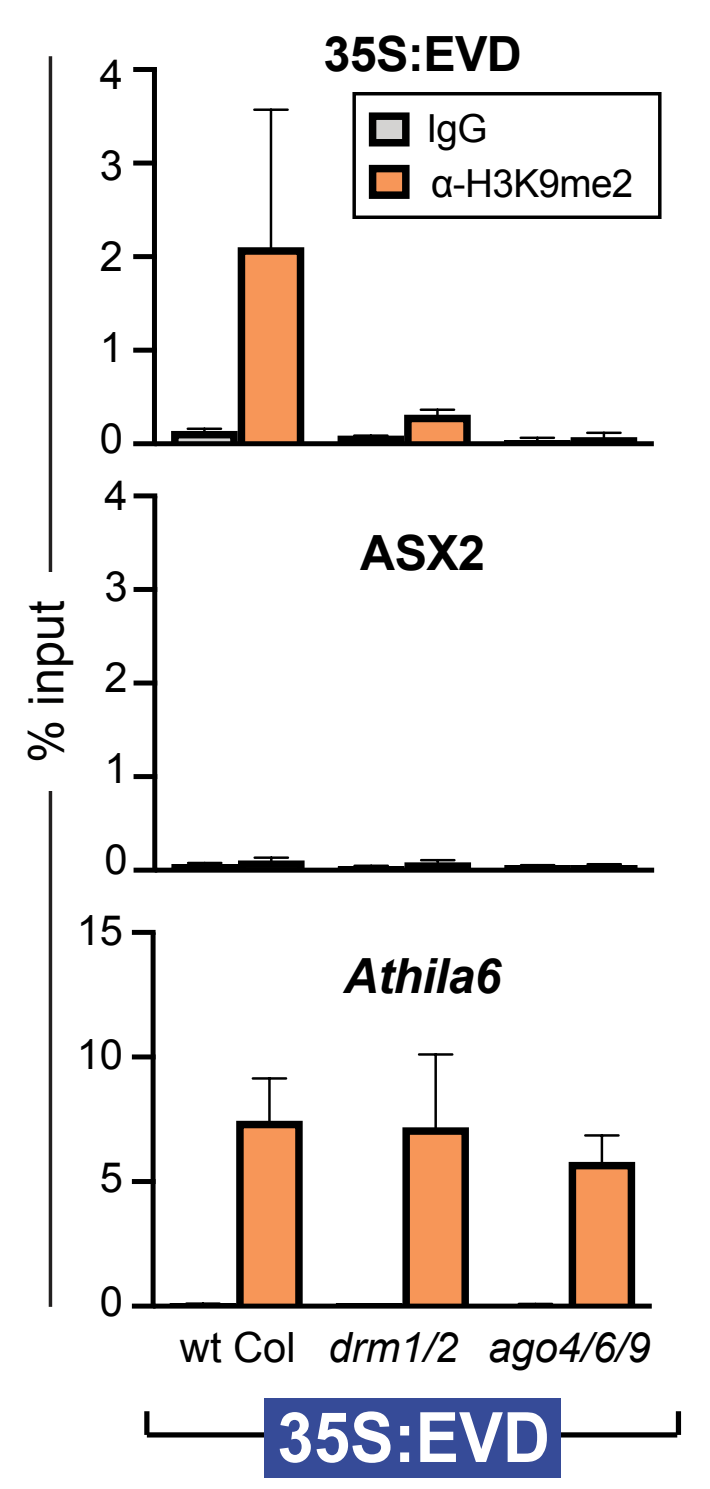

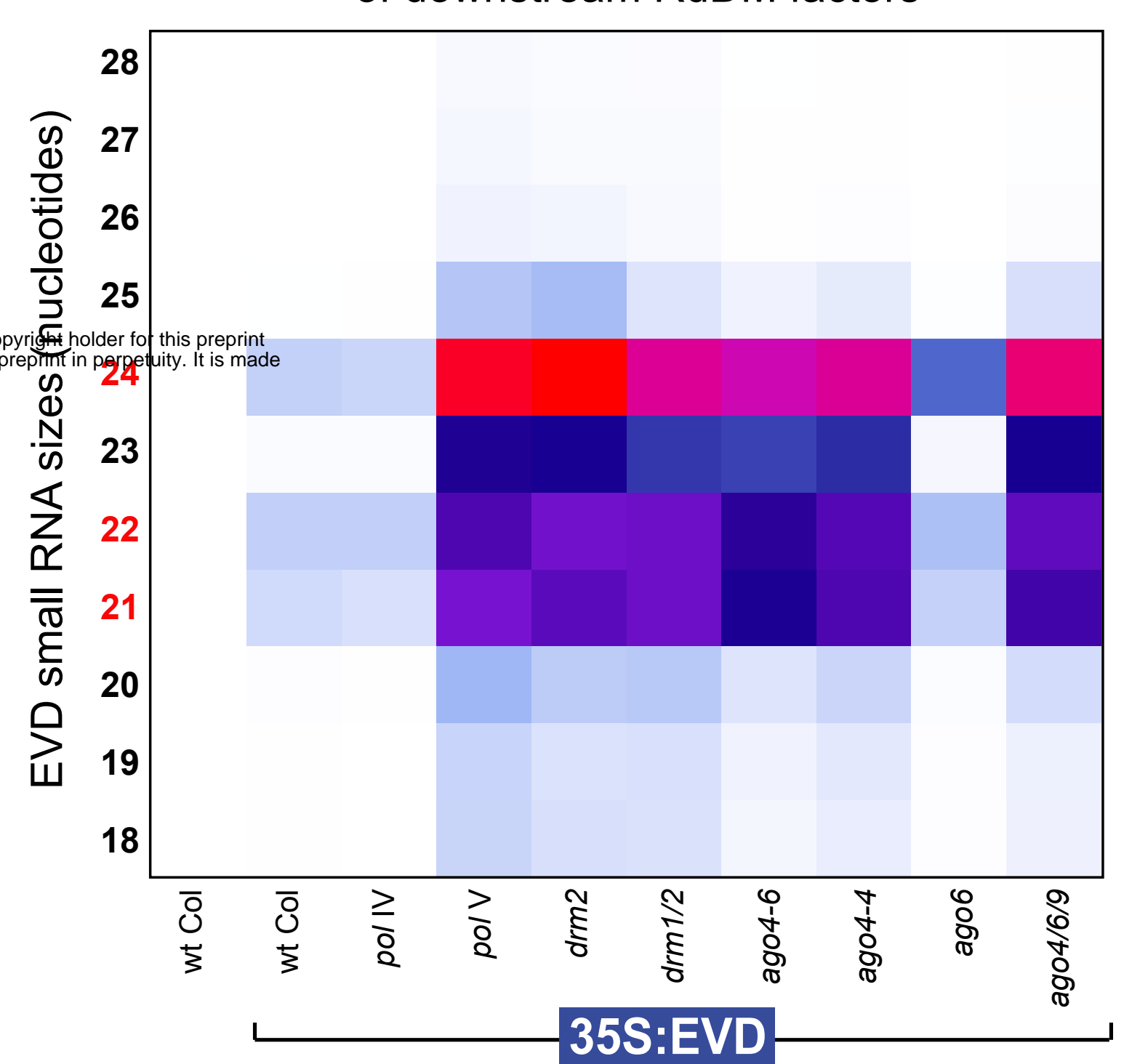

f DNA methylation in mutants of DCL proteins

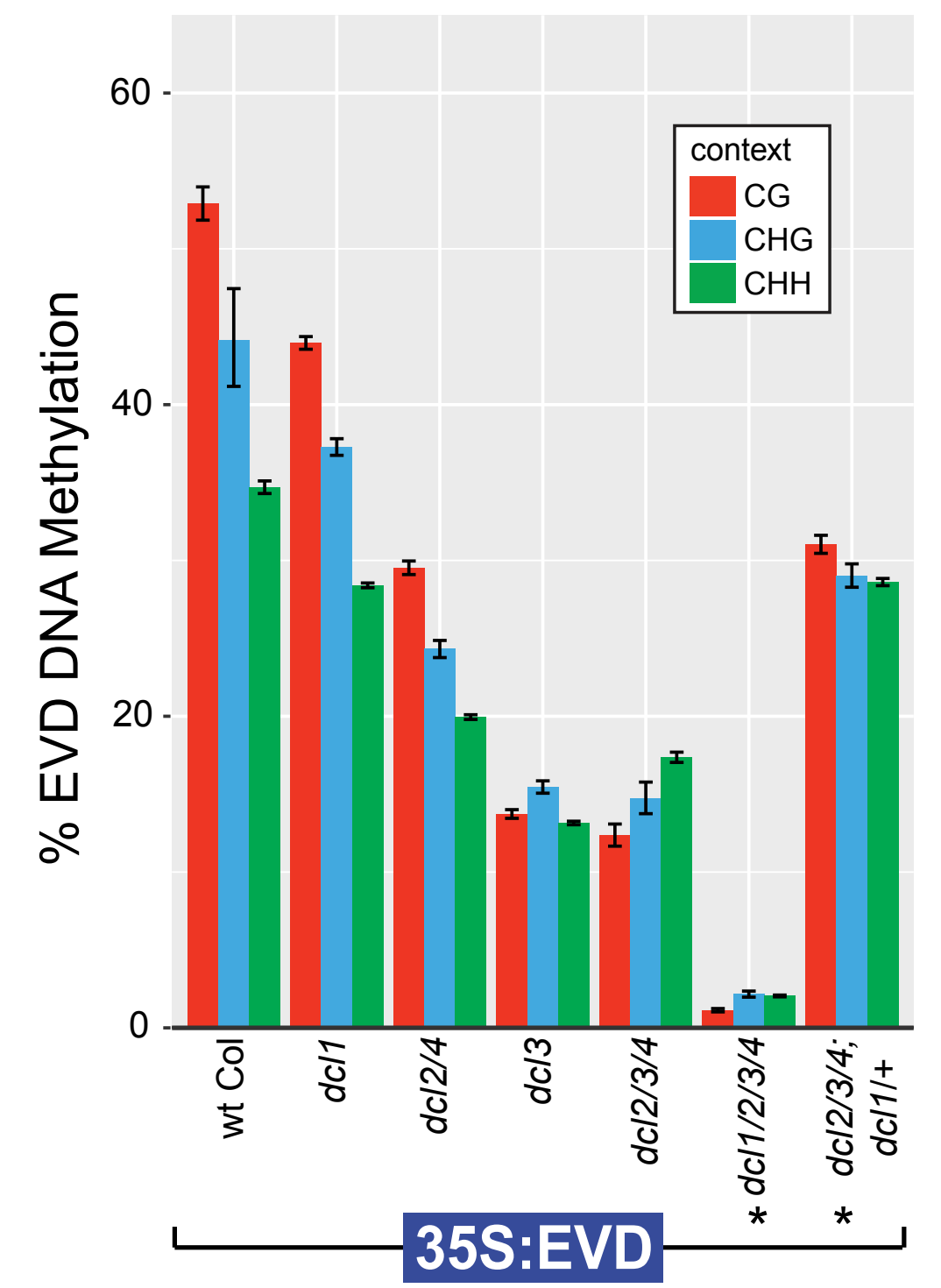

AGO4 incorporation of

EVD small RNAs
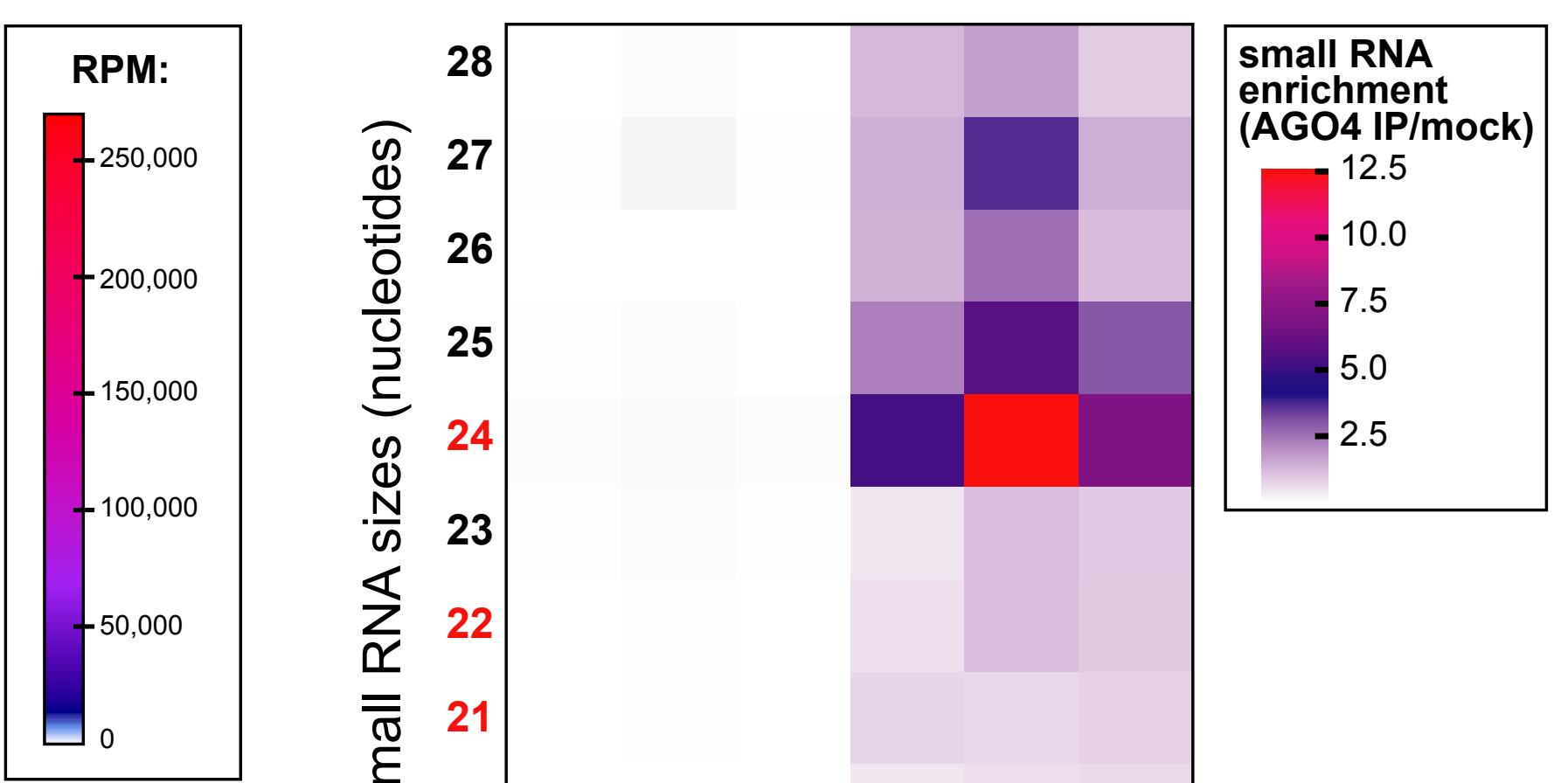

$<$

$\sum_{\simeq} 22$

$\overline{\bar{\sigma}} 21$

क 20

要 19

18

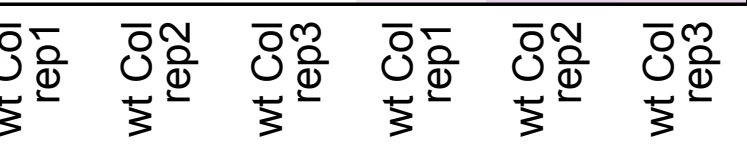

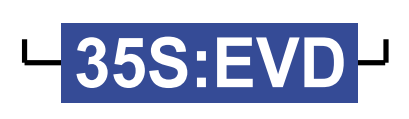

g Small RNA-seq in mutants

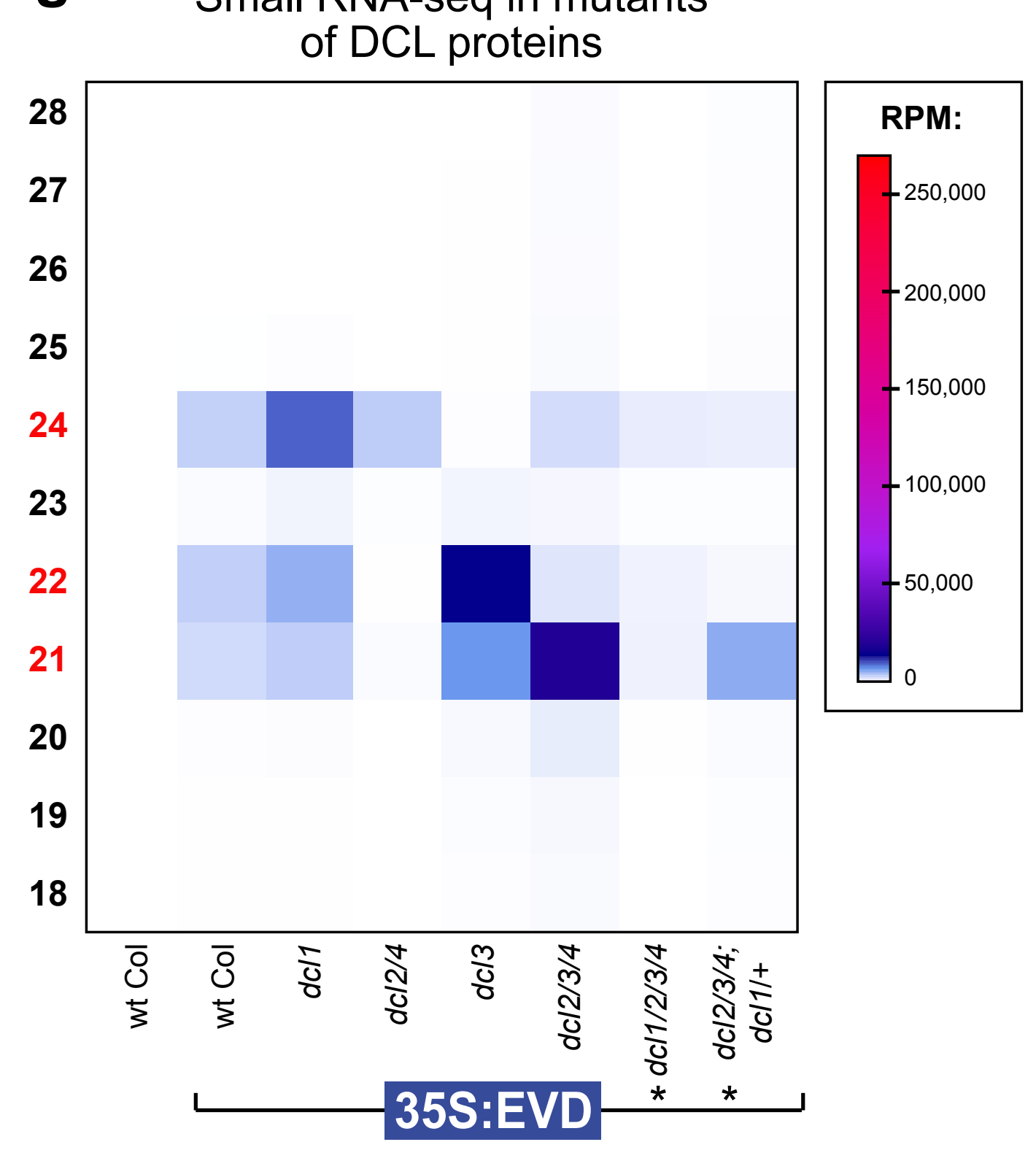




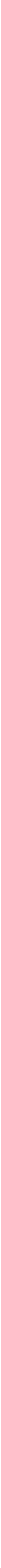


a

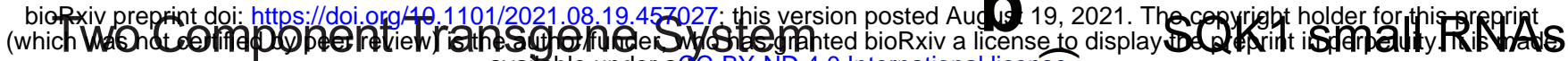
SQK1-IR Transgene avaliable under aCC-BY-ND 4.0 International licenis?

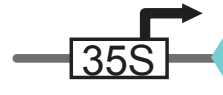

Targeting $\sqrt{=}=$ Diced $\stackrel{\text { Hairpin }}{=}$

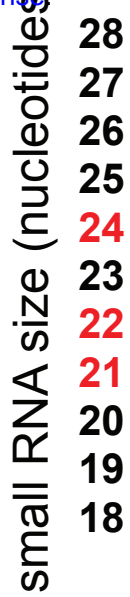

SQK1

IR Region

BSAS Amplicon

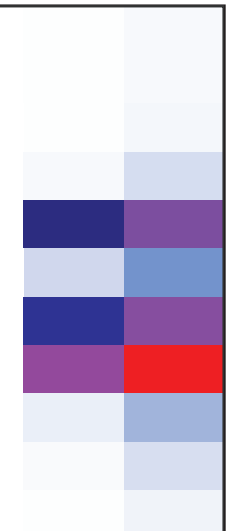

RPM:

TSS ATG

\section{SQK1 DNA Methylation}

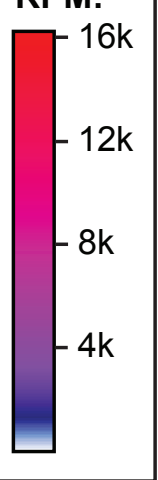

$50-$

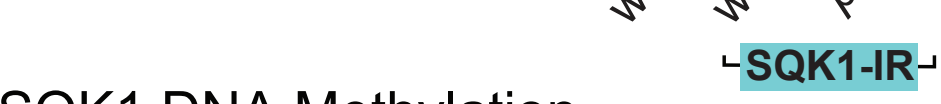

-SQK1-IR -

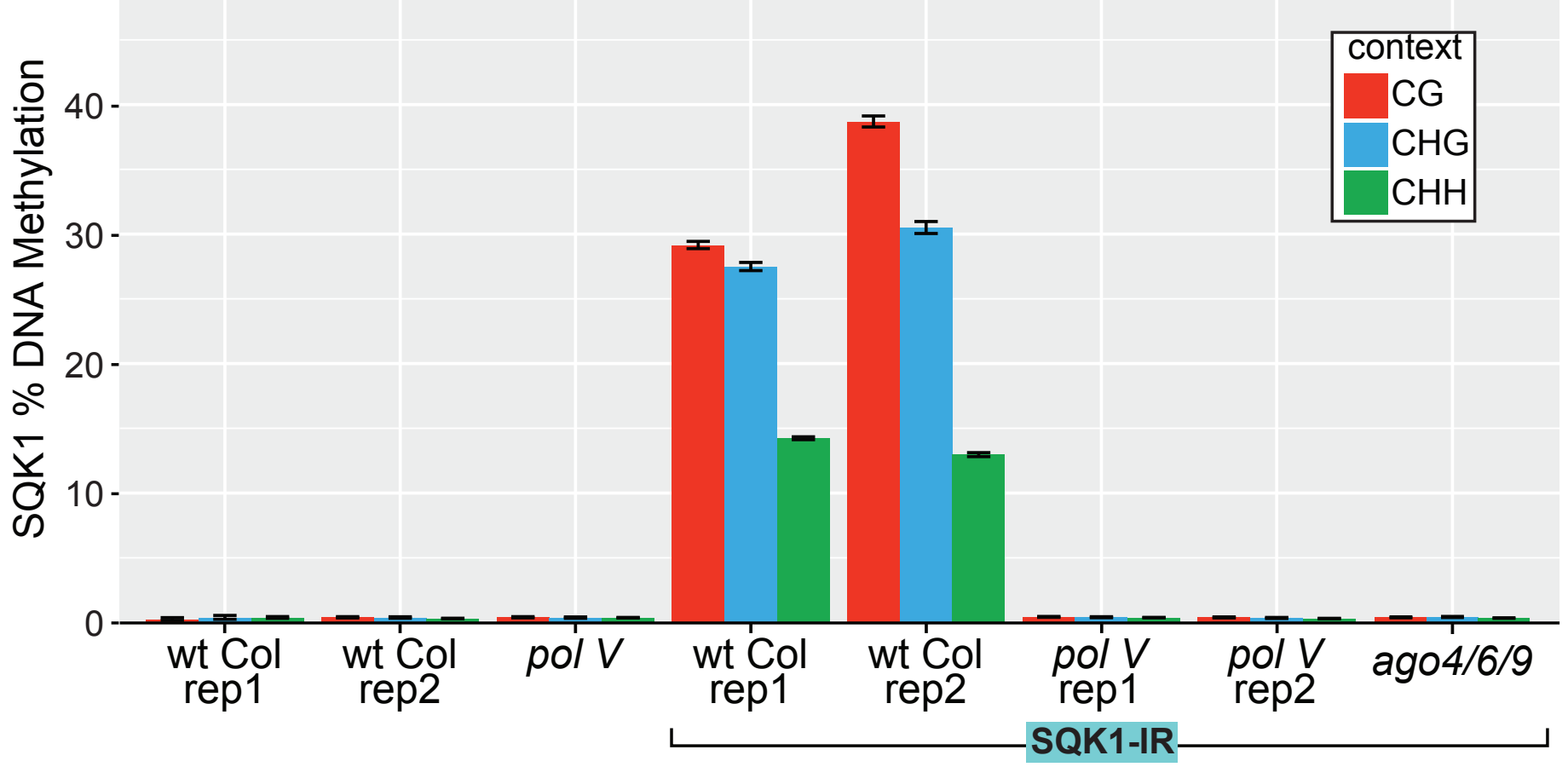

SQK1 RdDM Strength

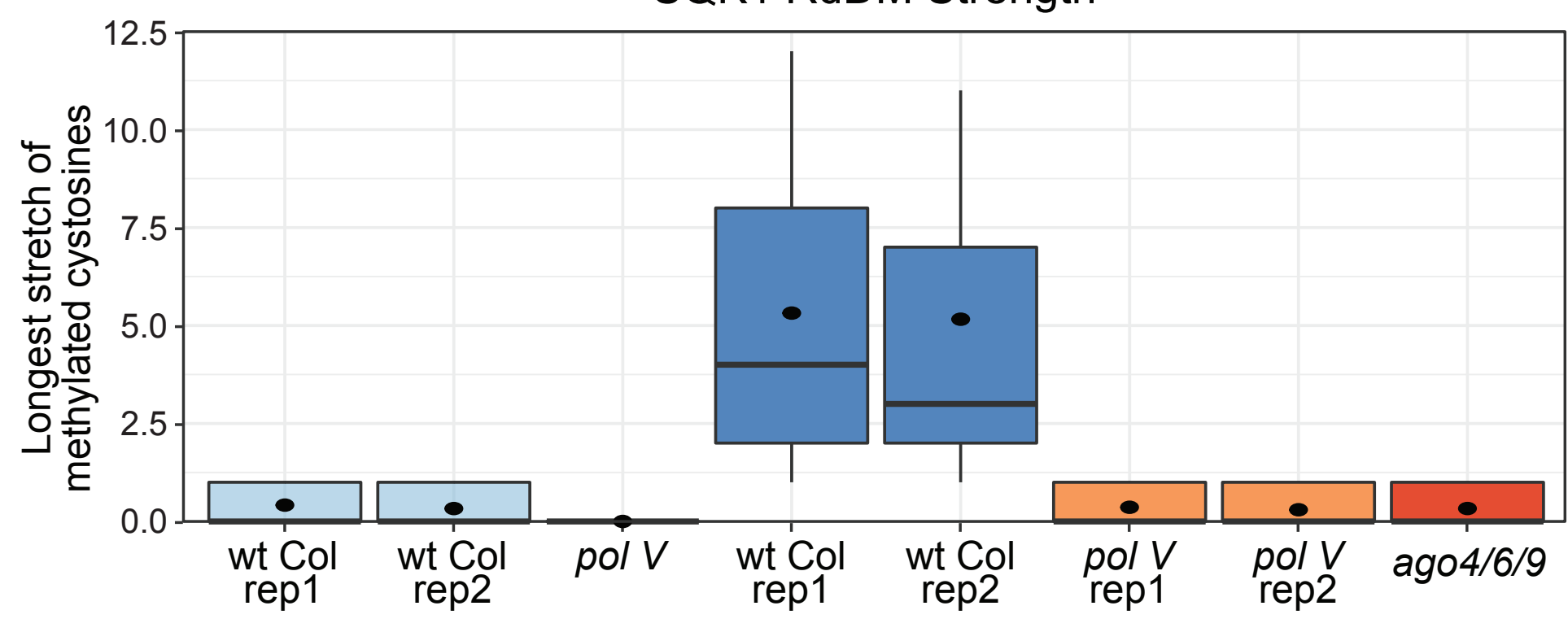




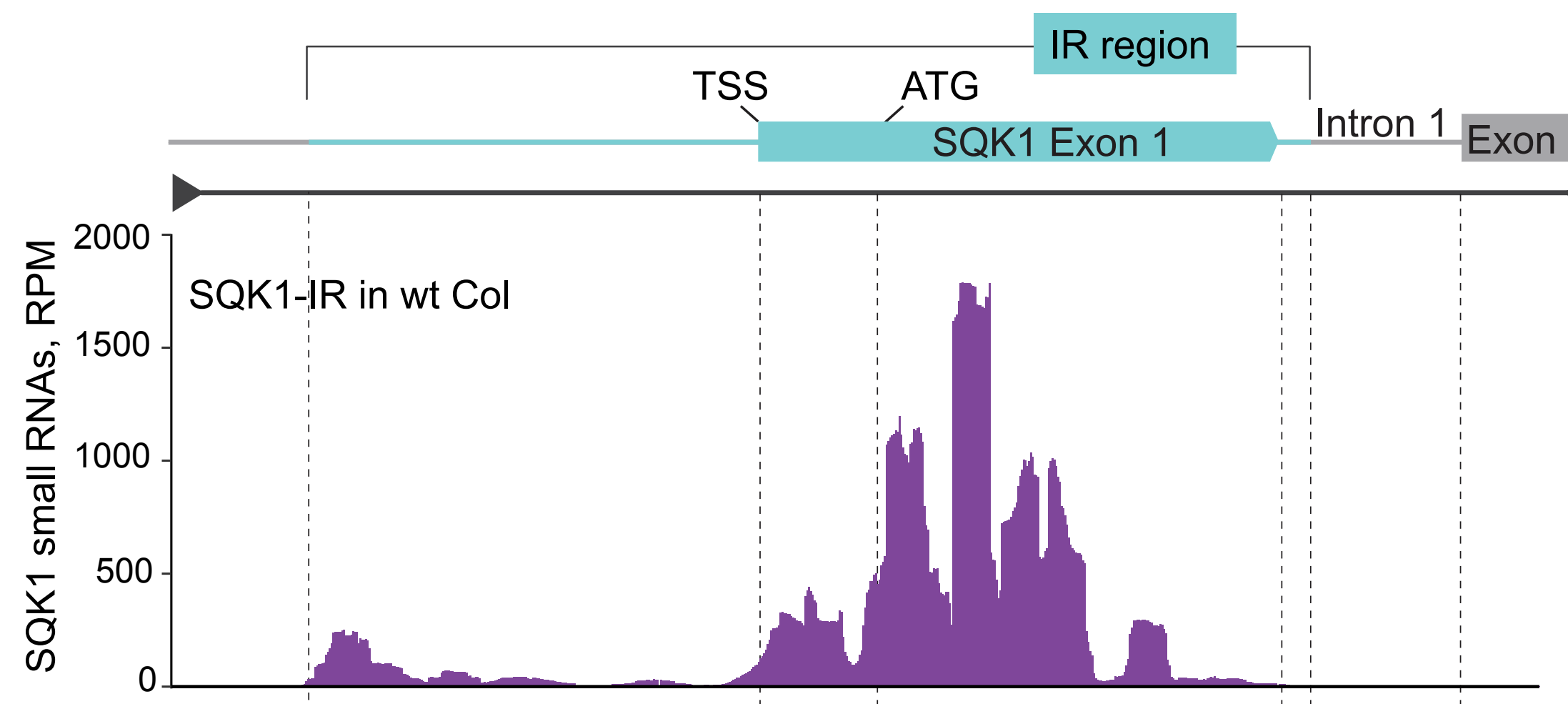

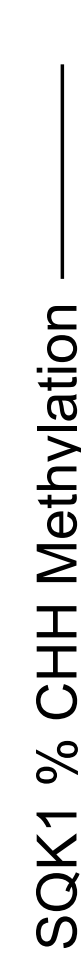

${ }^{75}$ SQK1-IR in wt Co

50

25.

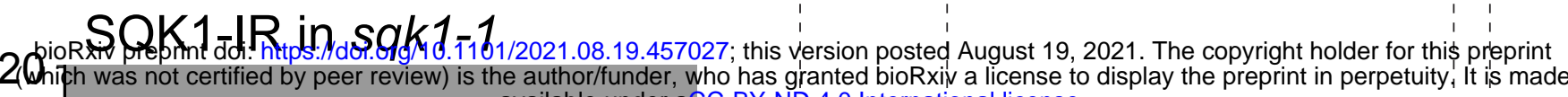

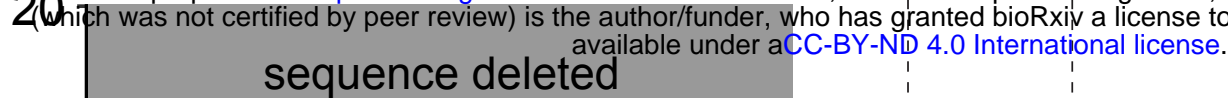

SQK1-IR + dCas9 in sqk1-1

20 sequence deleted

dCais9 gRNA d

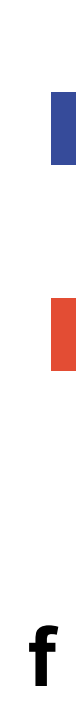

f 

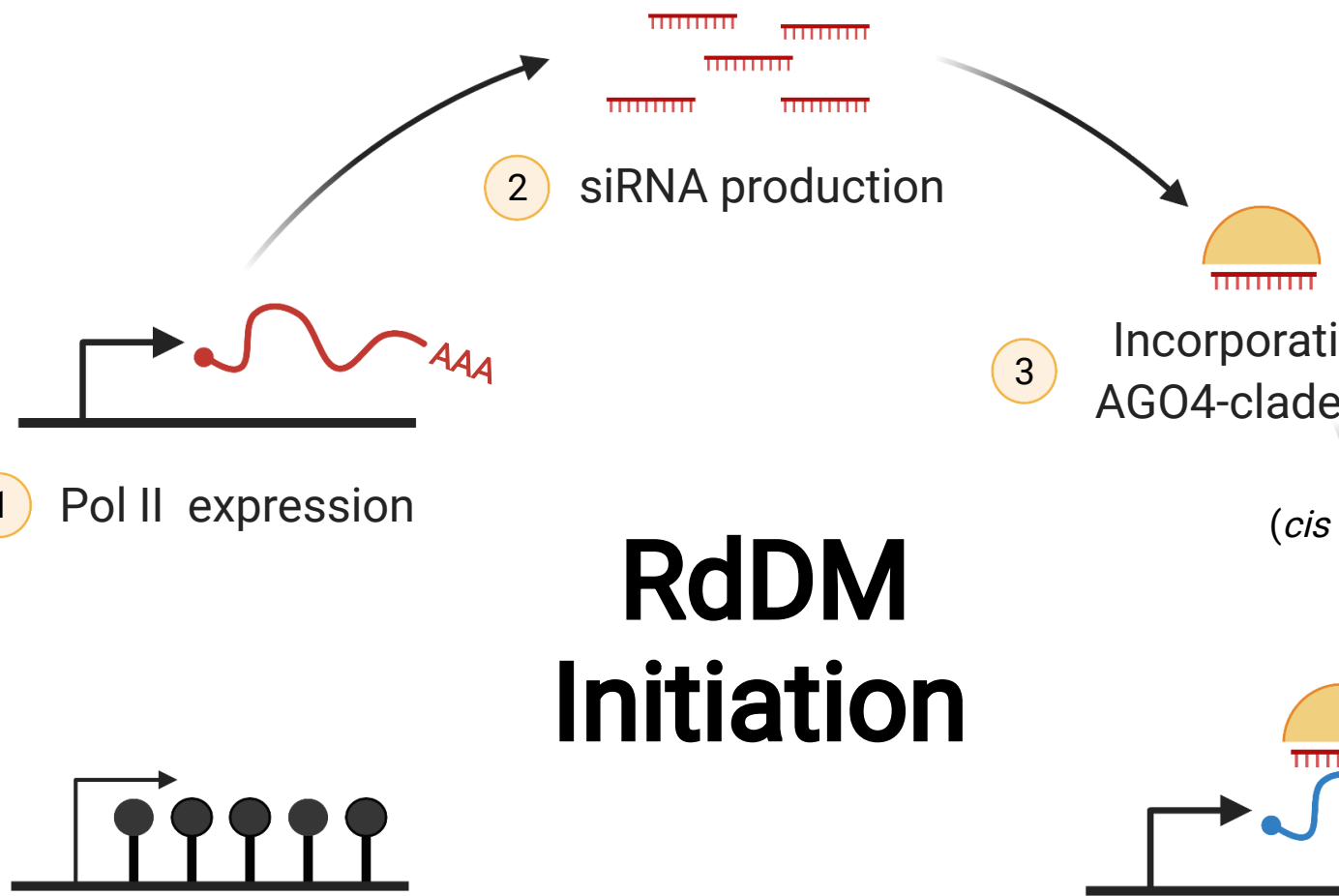

3 Incorporation into AG04-clade protein

1 Pol II expression

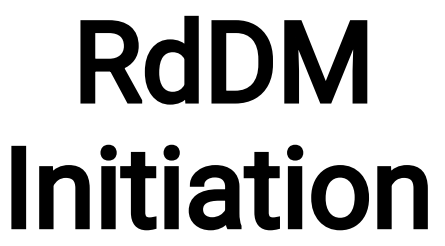 \\ RdDM} (cis or trans)

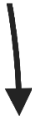

6 DNA methylation
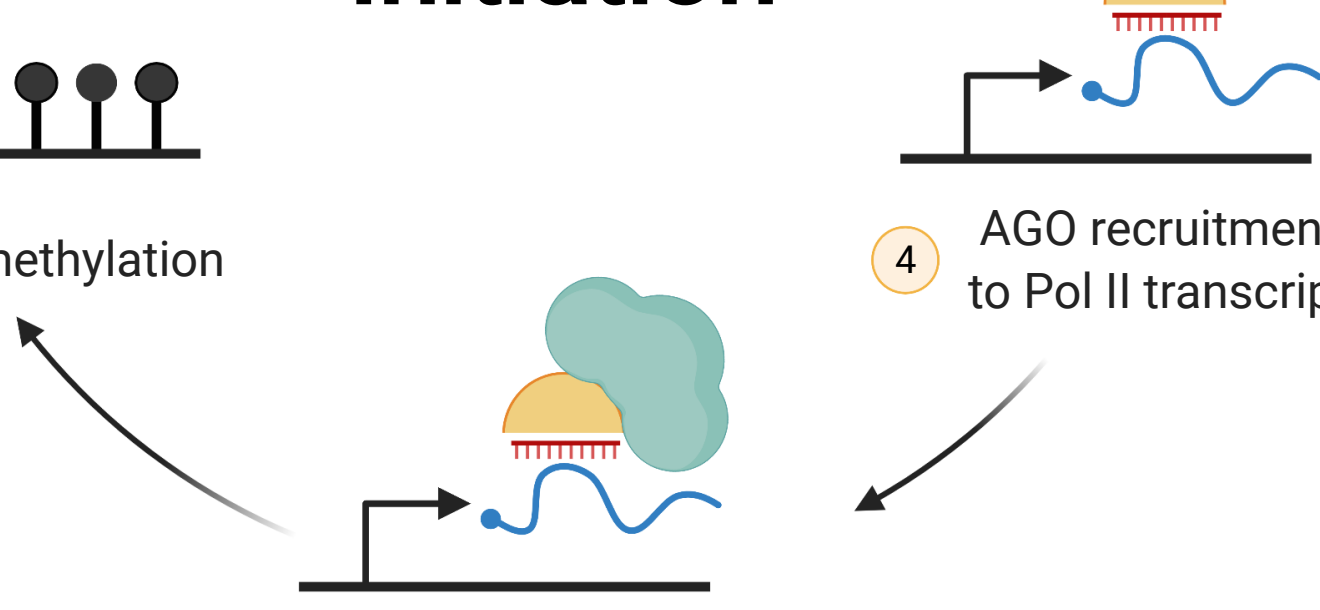

AGO recruitment
to Pol II transcript

5 Pol V recruitment 